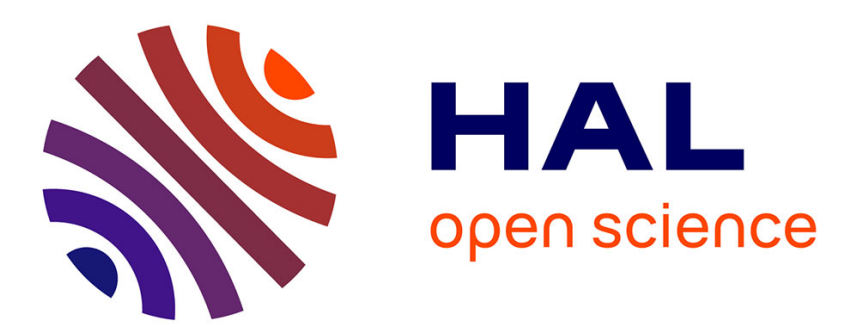

\title{
A hybrid stochastic/fixed-sectional method for solving the population balance equation
}

Alexandre Bouaniche, Luc Vervisch, Pascale Domingo

\section{To cite this version:}

Alexandre Bouaniche, Luc Vervisch, Pascale Domingo. A hybrid stochastic/fixed-sectional method for solving the population balance equation. Chemical Engineering Science, 2019, 209, pp.115198. 10.1016/j.ces.2019.115198 . hal-02313897

\section{HAL Id: hal-02313897}

\section{https://hal-normandie-univ.archives-ouvertes.fr/hal-02313897}

Submitted on 4 Dec 2020

HAL is a multi-disciplinary open access archive for the deposit and dissemination of scientific research documents, whether they are published or not. The documents may come from teaching and research institutions in France or abroad, or from public or private research centers.
L'archive ouverte pluridisciplinaire HAL, est destinée au dépôt et à la diffusion de documents scientifiques de niveau recherche, publiés ou non, émanant des établissements d'enseignement et de recherche français ou étrangers, des laboratoires publics ou privés. 


\title{
A hybrid Stochastic/Fixed-Sectional method for solving the Population Balance Equation
}

\author{
Alexandre Bouaniche ${ }^{\mathrm{a}}$, Luc Vervisch ${ }^{\mathrm{a}, *}$, Pascale Domingo ${ }^{\mathrm{a}}$ \\ ${ }^{a}$ CORIA - CNRS, Normandie Université, INSA de Rouen, Technopole du Madrillet, BP \\ 8, 76801 Saint-Etienne-du-Rouvray, France \\ Accepted for publication in Chemical Engineering Science
}

\begin{abstract}
The dynamics of flowing non-inertial particles undergoing nucleation, surface growth/loss, agglomeration and sometimes breakage, is usually characterised by the particle size distribution function. This distribution evolves according to a population balance equation. A novel approach combining Monte Carlo and fixed-sectional methods is proposed to minimise the discretisation errors when solving the surface growth/loss term of the population balance equation. The approach relies on a fixed number of stochastic particles and sections, with a numerical algorithm organised to minimise errors even for a moderate number of stochastic particles and sections. Canonical test cases featuring nucleation, agglomeration, and surface growth/loss are simulated. Results against the analytical solutions confirm the improvement in accuracy of the novel approach compared with fixed-sectional methods for the same computational effort. The hybrid method is thus of particular interest for simulating problems where surface growth/loss dominates the particles physics.
\end{abstract}

Keywords: Aerosol modelling; Sectional method; Stochastic method;

Hybrid modeling; Particle Size Distribution; Population Balance Equation, Probability Density Function, Monte Carlo solution

\footnotetext{
*Corresponding author

Email address: vervisch@coria.fr (Luc Vervisch)
} 


\section{Nomenclature}

$\begin{array}{ll}v & \text { particle characteristic size } \\ n(v ; \underline{x}, t) & \text { particle number density of size } v \text { per unit size } \\ N_{T}(\underline{x}, t) & \text { total number particle density } \\ \bar{P}\left(v^{*} ; \underline{x}, t\right) & \text { probability density function of particle size } \\ G(v) & \text { particle growth/loss rate } \\ \dot{H}_{o}(\underline{x}, t) & \text { nucleation source per unit of flow volume } \\ M & \text { number of sections } \\ v_{i} & \text { representative size of the } i \text {-th section of size } \\ N_{i}(\underline{x}, t) & \text { particle number density in the } i \text {-th section of size } \\ N_{i}^{R}(\underline{x}, t) & \text { residual particle number density in the } i \text {-th section of size } \\ \dot{A}_{i}(\underline{x}, t) & \text { agglomeration source in the } i \text {-th section of size } \\ \dot{A}_{T}(\underline{x}, t) & \text { total agglomeration source } \\ N_{P} & \text { total number of stochastic particles } \\ n_{P_{i}}(\underline{x}, t) & \text { number of stochastic particles in the } i \text {-th section of size }\end{array}$

\section{Introduction}

The numerical simulation of the dynamics of non-inertial particles in complex flows constitutes a real challenge. In many engineering processes, these particles nucleate, their size can grow or be reduced by surface chemical reactions and, while they are transported by the flow, they can agglomerate, or even break. Aside from the modeling of the complex physical and chemical phenomena at play, the numerics behind the simulation of these flows, which may operate in the turbulent regime, raises numerous issues. Background and comprehensive reviews on these subjects may be found in Ramkrishna (2000); Fox (2003); Marchisio \& Fox (2007) and references therein. Along these lines, a large variety of numerical approaches have been discussed in the literature to simulate crystallisation in liquid (Qamar et al., 2007), carbon and soot formation in flames (Leung et al., 1991; Balthasar \& Kraft, 2003; Ma et al., 2005; Lindstedt \& Louloudi, 2005; Zucca et al., 2006; Patterson \& Kraft, 2007; Eberle et al., 2017; Sewerin \& Rigopoulos, 2017; Rodrigues et al., 2018; Aubagnac-Karkar et al., 2018; Schiener \& Lindstedt, 2019; Franzelli et al., 2019) and many other chemical engineering applications with noninertial particles. Works have focused on numerical methods for the direct solving of the particle size distribution after discretisation of the phenomena driving its time evolution Gelbard \& Seinfeld, 1978; Hounslow et al. 
1988; Lister et al., 1995; Kumar \& Ramkrishna, 1996a b, 1997; Rigopoulos \& Jones, 2003; Filbet \& Laurenot, 2004; Park \& Rogak, 2004; Qamar et al., the problems from moments of the distribution (Frenklach, 2002; Mueller et al., 2009; Salenbauch et al., 2019).

The description of these complex multi-phase flows is usually tackled with a statistical formalism. The particle size distribution (PSD) function is inunit size and per unit flow volume. The time evolution of the PSD is governed by a Population Balance Equation (PBE) (Ramkrishna, 1985, 2000; Solsvik \& Jakobsen, 2015). Aside from the usual unsteady and flow convective terms, the PBE contains sources and sinks due to nucleation, agglomeration (Smolu(Das, 2016). In addition, because the particles sizes can increase or decrease by surface reaction, thus without change of the number of particles, a conservative convective flux in size space also appears in the PBE (Hulburt \& Katz, 1964).

As for any non-linear convective effect (Ferziger \& Perić, 1996), a stable numerical discretisation of this surface growth/loss term results from a compromise between stability and numerical diffusion. In the case of physical problems for which nucleation is associated to fast agglomeration, the error accumulated in the PSD when solving for particles surface change may not be a serious issue. However, focussing on specific physical phenomena, or cal kinetics or other models against experiments requires a precise treatment. This is particularly the case in the careful validation of the basic mechanisms driving sooting flames (Desgroux et al., 2017), or more generally in any flow where agglomeration is slower than surface growth or loss, as previously discussed by Park \& Rogak (2004).

As noticed above, many numerical approaches already exist and some have been specifically developed with success to minimise numerical errors associated to particles surface evolution (Kumar \& Ramkrishna, 1996a b, 1997; Tsantilis et al., 2002; Park \& Rogak, 2004; Nguyen et al., 2016; Sewalong these lines, by discussing an alternative strategy to solve for particles growth/loss in the PBE.

The PSD, number of particles per unit of flow volume and per unit of particle size, is first decomposed into the total number of particles per unit 
particle size. The total number of particles per unit of flow volume varies with nucleation and a sink due to agglomeration or disappearance. The transport equation for the probability density function of size is then derived from the population balance equation of the PSD. A dual discretisation of size space 65 is proposed, based on both fixed sections (i.e., sections boundaries are fixed) and a fixed number of stochastic particles. The sections of size are defined as in well established fixed-sectional methods and allow for computing the amplitude of the agglomeration and nucleation sources from physical modeling. Each stochastic particle carries information on size independently of sections, a distribution of sizes over the stochastic particles representative of the PDF. Growth is then applied to every stochastic particle in the form of a simple linear problem, free from any artificial diffusion in size space.

A Monte Carlo procedure with random selection and movement of the stochastic particles between sections is designed to simulate agglomeration and nucleation. The number of particles selected is calibrated by the sources and sinks computed over the sections. Because the number of stochastic particles stays finite, a residual exists in the form of a roundoff error between the exact continuous PSD and the distribution reconstructed from the PDF discretised over the stochastic particles. To overcome this issue, the evolution of this residual is solved with a fixed-sectional method and cumulated till the roundoff error becomes large enough to be redistributed over a finite number of stochastic particles.

Using a moderate number of stochastic particles, the CPU time stays of the order of the one required by fixed-sectional methods, but particles surface change (growth/loss) is solved with a very limited amount of numerical diffusion of the PSD in size space.

The hybrid sectional Monte Carlo solution discussed in this work stands as a numerical method to solve for a PDF balance equation. It should not be confused with direct Monte Carlo solutions of non-inertial particles dynamics, 90 which aim at performing a direct numerical simulation of the elementary physical phenomena acting on the particles (Falope et al., 2001; Li et al., 2001; Oullion et al., 2009; Pesmazoglou et al., 2016). The presented method also differs from constant number Monte Carlo methods (Smith \& Matsoukas, 1998) or differentially weighted Monte Carlo approaches (Lee et al., 2015; Patterson et al., 2011; Zhao \& Zheng, 2013). In these methods, collision frequencies and corresponding agglomeration source terms are calculated over all stochastic particles or strategies like majorant kernels are proposed to avoid calculating collision probabilities between all stochastic particles. CPU 
cost depends mainly on the number of stochastic particles in these former methods. In the proposed hybrid approach, agglomeration source terms are calculated from the particle number density discretised over the sections, as in fixed-sectional methods and therefore for a much lower CPU cost (which depends primarily on the number of sections), allowing a future application to the simulation of real systems.

The paper is organised as follows, the formulation of the problem is first given in the subsequent section. Then, the attempt to set up a hybrid stochastic/fixed-sectional approach is reported in detail. A set of wellestablished canonical test cases retained for evaluating the method are presented before discussing the results obtained. Time evolutions of PSD are compared with the analytical solutions and simulations using fixed-sectional methods. Discretisation errors are quantified from the moments and the Wasserstein metric of size distributions. Convergence and response to the resolution parameters are also studied.

\section{Problem formulation}

The Particle Size Distribution $n(v ; \underline{x}, t)$, number of particles of characteristic size $v$ (in terms of volume or mass, $v$ is a continuous independent variable), per unit of flow volume and per unit of characteristic size of an aerosol submitted to simultaneous nucleation, surface variation and agglomeration, is governed by a Population Balance Equation (PBE) (Ramkrishna, 2000; Solsvik \& Jakobsen, 2015):

$$
\begin{gathered}
\frac{\partial n(v ; \underline{x}, t)}{\partial t}+\mathbf{u} \cdot \nabla n(v ; \underline{x}, t)+\frac{\partial}{\partial v}[G(v) n(v ; \underline{x}, t)]=\dot{h}\left(v_{o} ; \underline{x}, t\right) \\
+\frac{1}{2} \int_{0}^{v} \beta(v-\bar{v}, \bar{v}) n(v-\bar{v} ; \underline{x}, t) n(\bar{v} ; \underline{x}, t) d \bar{v}-n(v ; \underline{x}, t) \int_{0}^{\infty} \beta(v, \bar{v}) n(\bar{v} ; \underline{x}, t) d \bar{v}
\end{gathered}
$$

where usual notations are adopted. $G(v)>0$ is the surface growth rate or $G(v)<0$ the surface loss rate. $\dot{h}\left(v_{o}\right)>0$ is the nucleation rate or $\dot{h}\left(v_{o}\right)<0$ the disappearance rate, seen at size $v_{o}$. The integral source term on the RHS accounts for agglomeration following the continuous counterpart of Smoluchowski equation (Smoluchowski, 1917), with $\beta(v, \bar{v})$ the collision kernel for two particles of volume $v$ and $\bar{v}$. The PSD evolution is thus driven by an integro-partial-differential equation of the hyperbolic type.

The surface variation rate $G(v)$ stands as a convective term in the particle size space. Resolution of $G(v)$ is challenging, similarly to the non-linear flow 
convective term in physical space (Ferziger \& Perić, 1996), which motivates 125 the present study.

Further quantities related to the PSD are usually introduced. $N_{i}(\underline{x}, t)$ is defined as the number of particles of characteristic size $v_{i}$ per unit of flow volume

$$
N_{i}(\underline{x}, t)=\int_{I_{v_{i}}} n(v ; \underline{x}, t) d v,
$$

where the interval $I_{v_{i}} \equiv\left[v_{i}^{i n f}, v_{i}^{\text {sup }}\right]$ defines the $i$-th fixed-section of size. The total number density per unit of flow volume is the sum over all sizes or over the $M$ sections considered

$$
N_{T}(\underline{x}, t)=\int_{v_{o}}^{\infty} n(v ; \underline{x}, t) d v=\sum_{i=1}^{M} N_{i}(\underline{x}, t) .
$$

Similarly, the nucleation source per unit of flow volume is

$$
\dot{H}_{o}(\underline{x}, t)=\int_{I_{v_{o}}} \dot{h}(v ; \underline{x}, t) d v .
$$

The Smoluchowski agglomeration sources/sink (Eq. (1)),

$$
\begin{aligned}
& \dot{a}(v ; \underline{x}, t) \\
& =\frac{1}{2} \int_{0}^{v} \beta(v-\bar{v}, \bar{v}) n(v-\bar{v} ; \underline{x}, t) n(\bar{v} ; \underline{x}, t) d \bar{v}-n(v ; \underline{x}, t) \int_{0}^{\infty} \beta(v, \bar{v}) n(\bar{v} ; \underline{x}, t) d \bar{v},
\end{aligned}
$$

leads to the definition of the agglomeration source for the $i$-th section

$$
\dot{A}_{i}(\underline{x}, t)=\int_{I_{v_{i}}} \dot{a}(v ; \underline{x}, t) d v,
$$

and $A_{T}$ is the total sink due to agglomeration over all particles, thus the sum 
of $A_{i}(\underline{x}, t)$ over all sections

$$
\dot{A}_{T}(\underline{x}, t)=\int_{v_{o}}^{\infty} \dot{a}(v ; \underline{x}, t) d v=\sum_{i=1}^{M} \dot{A}_{i}(\underline{x}, t) .
$$

All these quantities allow for combining the PBE with the evolution of the probability density function of the characteristic particle size.

\section{Hybrid Stochastic/Fixed-Sectional method}

\subsection{Control parameters and statistical description}

To benefit from a description in which surface growth or loss is cast into the form of a linear term, instead of directly solving for the population balance equation, it is proposed to consider both $N_{T}(\underline{x}, t)$, the total number of particles per unit volume, and $\bar{P}\left(v^{*} ; \underline{x}, t\right)$, the probability density function (PDF) of the particles characteristic size, where $v^{*} \in\left[v_{o}, \infty\right]$ denotes the sample space variable associated to $v$, seen as a random variable.

The relation between $n(v ; \underline{x}, t)$, the particle number density per unit size, $N_{i}(\underline{x}, t)$, the number density of particles whose size is in the section $I_{v_{i}}(v \in$ $\left.I_{v_{i}}\right)$ at the flow position ' $\underline{x}$ ' at time ' $\mathrm{t}$ ' (Eq. (2)), and $\bar{P}\left(v^{*} ; \underline{x}, t\right)$, the PDF of the particles sizes reads:

$$
\int_{I_{v_{i}}} n\left(v^{*} ; \underline{x}, t\right) d v^{*}=N_{i}(\underline{x}, t)=N_{T}(\underline{x}, t) \int_{I_{v_{i}}} \bar{P}\left(v^{*} ; \underline{x}, t\right) d v^{*},
$$

where

$$
\int_{I_{v_{i}}} \bar{P}\left(v^{*} ; \underline{x}, t\right) d v^{*}
$$

is the probability to find particles of sizes $v \in I_{v_{i}}$. Because (8) should be valid whatever $I_{v_{i}}$,

$$
n\left(v^{*} ; \underline{x}, t\right)=N_{T}(\underline{x}, t) \bar{P}\left(v^{*} ; \underline{x}, t\right) .
$$

The function

$$
\begin{aligned}
\delta\left(v-v^{*}\right) & =\lim _{d v \rightarrow 0} 1 / d v \text { if } v \in\left[v^{*}-d v / 2, v^{*}+d v / 2\right] \\
& =0 \text { otherwise }
\end{aligned}
$$


is introduced and $\bar{P}\left(v^{*} ; \underline{x}, t\right)=\overline{\delta\left(v(\underline{x}, t)-v^{*}\right)}$, where - denotes a statistical average (Lundgren, 1967; Dopazo, 1979; Kollmann, 1990; Dopazo et al., 1997).

${ }_{140}$ The nucleation term in the PBE (Eq. (1) ) may be written $\dot{h}\left(v_{o} ; \underline{x}, t\right)=$ $\dot{H}_{o}(\underline{x}, t) \delta\left(v_{o}-v^{*}\right)$, with $\dot{H}_{o}(\underline{x}, t)$ defined by $(4)$ in the limit where the size of the interval $I_{o}$ goes to zero. Similarly, the agglomeration term may be written $\dot{a}\left(v^{*} ; \underline{x}, t\right)=\dot{A}_{i}(\underline{x}, t) \delta\left(v_{i}-v^{*}\right)$, with $\dot{A}_{i}(\underline{x}, t)$ defined by $(6)$ in the limit where $I_{v_{i}}$ goes to zero. Then the PBE formally becomes

$$
\begin{aligned}
\frac{\partial n\left(v^{*} ; \underline{x}, t\right)}{\partial t}+\mathbf{u} \cdot \nabla n\left(v^{*} ; \underline{x}, t\right) & +\frac{\partial}{\partial v^{*}}\left[G\left(v^{*}\right) n\left(v^{*} ; \underline{x}, t\right)\right] \\
& =\dot{H}_{o}(\underline{x}, t) \delta\left(v_{o}-v^{*}\right)+\dot{A}_{i}(\underline{x}, t) \delta\left(v_{i}-v^{*}\right) .
\end{aligned}
$$

The total number density $N_{T}$ evolves according to

$$
\frac{\partial N_{T}(\underline{x}, t)}{\partial t}+\mathbf{u}(\underline{x}, t) \cdot \nabla N_{T}(\underline{x}, t)=\dot{H}\left(v_{o} ; \underline{x}, t\right)+\dot{A}_{T}(\underline{x}, t)
$$

with $\dot{A}_{T}(\underline{x}, t)$ given by (7). From 10 the PDF evolves as

$$
\frac{\partial \bar{P}\left(v^{*} ; \underline{x}, t\right)}{\partial t}=\left[\frac{1}{n\left(v^{*} ; \underline{x}, t\right)} \frac{\partial n\left(v^{*} ; \underline{x}, t\right)}{\partial t}-\frac{1}{N_{T}(\underline{x}, t)} \frac{\partial N_{T}(\underline{x}, t)}{\partial t}\right] \bar{P}\left(v^{*} ; \underline{x}, t\right) .
$$

Introducing $(13)$ and $(14)$ in this relation, the PDF evolution equation is obtained

$$
\begin{aligned}
& \frac{\partial \bar{P}\left(v^{*} ; \underline{x}, t\right)}{\partial t}+\mathbf{u}(\underline{x}, t) \cdot \nabla \bar{P}\left(v^{*} ; \underline{x}, t\right)=\overbrace{-\frac{\partial}{\partial v^{*}}\left[G\left(v^{*}\right) \bar{P}\left(v^{*} ; \underline{x}, t\right)\right]}^{(i)} \\
& +\underbrace{\frac{\dot{H}_{o}(\underline{x}, t)}{N_{T}(\underline{x}, t)}\left(\delta\left(v_{o}-v^{*}\right)-\bar{P}\left(v^{*} ; \underline{x}, t\right)\right)}_{(i i)} \\
& +\underbrace{\frac{1}{N_{T}(\underline{x}, t)}\left(\dot{A}_{i}(\underline{x}, t) \delta\left(v_{i}-v^{*}\right)-\dot{A}_{T}(\underline{x}, t) \bar{P}\left(v^{*} ; \underline{x}, t\right)\right)}_{(i i i)} .
\end{aligned}
$$

In this balance equation, as in the PBE, the change of particles sizes at the rate $G\left(v_{i}\right)$ is a convective term in size space (term $(i)$ ). The term $(i i)$ on the 
RHS is nucleation, which is decomposed into two parts preserving the normalisation of the PDF. The first, proportional to $\delta\left(v_{o}-v^{*}\right)$, increases the probability to find the smallest particles at the nucleation rate $\dot{H}_{o}(\underline{x}, t) / N_{T}(\underline{x}, t)$,

150 while the second decreases, at the same rate, the probability for all sizes. A similar formulation is found for agglomeration (term $(i i i)$ ), with the probability evolving at the positive or negative rate $\dot{A}_{i}(\underline{x}, t) / N_{T}(\underline{x}, t)$, associated to a correction proportional to $-\dot{A}_{T}(\underline{x}, t) / N_{T}(\underline{x}, t)>0$, so that the PDF normalisation is preserved. Indeed, when two particles of characteristic sizes $v_{i}$ and $v_{j}$ agglomerate, the probability of their respective initial size decreases $\left(\dot{A}_{i}(\underline{x}, t)<0\right.$ and $\left.\dot{A}_{j}(\underline{x}, t)<0\right)$, to increase the probability of their new size $v_{k}\left(\dot{A}_{k}(\underline{x}, t)>0\right)$. However, because the total number of physical particles decreases in this process, the probability of all sizes benefit from an increase proportional to $-A_{T}$, the overall particle sink.

160 The solutions of the equations (14) and (16) provide all the necessary information to simulate the nucleation and the growth of an ensemble of particles transported in a flow. The particle size distribution $N_{i}(\underline{x}, t)$ can then be recovered from (8).

Because the focus is on the numerical solving of terms controlling the PSD shape, a perfectly stirred/homogeneous reactor is considered $(\mathbf{u}=0)$. However, the straightforward addition of the convective flow velocity will be discussed in the conclusion.

\subsection{Hybrid Stochastic/Fixed-Sectional solution}

The probability density function $\bar{P}\left(v^{*} ; t\right)$ can be discretised over a set of $N_{P}$ stochastic particles ${ }^{1}$ each carrying information on the particle size, i.e. $v=v^{k}$ for $k=1, \cdots, N_{P}$ and $\bar{P}\left(v^{*} ; t\right)=\left(1 / N_{P}\right) \sum_{k=1}^{N_{P}} \delta\left(v^{k}(t)-v^{*}\right)$. The total number of stochastic particles $N_{P}$ is fixed.

The $v$-space is also discretised in $M$ fixed sections, to define a mesh providing a distribution of $\Delta v_{i}=v_{i}^{\text {sup }}-v_{i}^{\text {inf }}$, for $i=1, \cdots, M$. Uniform, geometric and exponential sectional grids will be tested thereafter. The characteristic size $v^{k}$ of a stochastic particle can take any value between the considered size bounds $\left[v_{o}, v_{M}\right]$, independently of the fixed sectional mesh.

Within this set of $N_{P}$ particles, an integer number $n_{P_{i}}(t)$ of stochastic particles features sizes so that $v^{k} \in I_{v_{i}} \equiv\left[v_{i}^{\text {inf }}, v_{i}^{\text {sup }}\right]$. This number of stochastic particles relates to the PDF and to $N_{i}(t)$, the number densities of the physical

\footnotetext{
${ }^{1}$ Space is omitted in this subsection for brevity.
} 
particles (Eq. (8)), according to:

$$
\int_{I_{v_{i}}} \bar{P}\left(v^{*} ; t\right) d v^{*}=\frac{n_{P_{i}}(t)}{N_{P}}=\frac{N_{i}(t)}{N_{T}(t)} .
$$

To simulate the PDF time evolution through $v^{k}(t)$, the stochastic particles time evolution, a fractional-step method is followed. Starting at time $t^{n}$, surface growth/loss is first applied to advance the solution to time $t^{n+\frac{1}{2}}=$ $t^{n}+\delta t / 2$. This is applied in a deterministic way to every $k$-th particle, as a simple linear process proportional to $G\left(v^{k}(t)\right)$, which is the major advantage of the proposed approach. Then from the time $t^{n+\frac{1}{2}}$, the solution is advanced to $t^{n+1}=t^{n+\frac{1}{2}}+\delta t / 2$ by applying nucleation and agglomeration effects, which are simulated by moving the stochastic particles between the defined sections. The number of stochastic particles randomly selected to be removed from a section and dispatched over the others, are calculated according to the nucleation and agglomeration rates controlling the PDF evolution (Eq. (16)). At every instant $t^{n}, \delta t$ is determined so that stability is secured, different amplitudes of $\delta t$ may be required in practice to advance from $t^{n}$ to $t^{n+\frac{1}{2}}$ (growth/loss) and from $t^{n+\frac{1}{2}}$ to $t^{n+1}$ (nucleation and agglomeration).

\subsubsection{Surface growth/loss}

During surface growth or loss, the size of the $k$-th stochastic particle evolves according to:

$$
\frac{d v^{k}(t)}{d t}=G\left(v^{k}(t)\right), \quad k=1, \cdots, N_{P}
$$

Each stochastic particle then carries information on an updated size $v^{k}\left(t^{n+\frac{1}{2}}\right)$. The total number density stays constant during growth $\left(d N_{T}(t) / d t=0\right)$. stochastic particles is available and the $\operatorname{PDF} \bar{P}\left(v^{*} ; t^{n+\frac{1}{2}}\right)$ is known along with $n_{P_{i}}\left(t^{n+\frac{1}{2}}\right)$ the number of stochastic particles in every section.

\subsubsection{Nucleation and agglomeration}

Nucleation and agglomeration are subsequently applied, which impacts on the number density $N_{T}(t)$ and on the PDF through the change of $n_{P_{i}}\left(t^{n+\frac{1}{2}}\right)$ for each interval $I_{v_{i}}$. Starting from $N_{T}\left(t^{n}\right)=N_{T}\left(t^{n+\frac{1}{2}}\right)$, the number density 


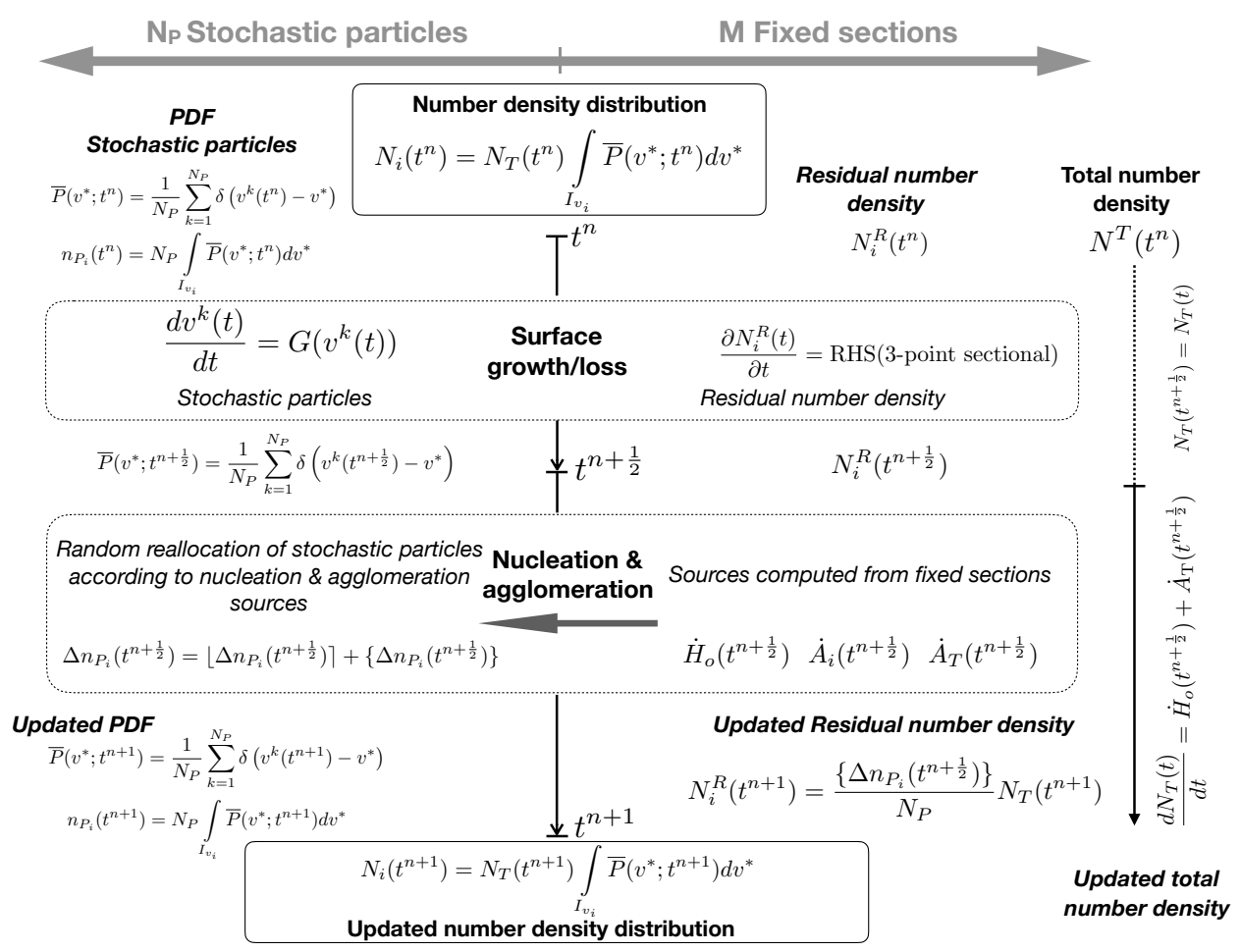

Figure 1: Flowchart of the hybrid stochastic/fixed-sectional method.

evolves from $t^{n+\frac{1}{2}}$ to $t^{n+1}$ with

$$
\frac{d N_{T}(t)}{d t}=\dot{H}_{o}(t)+\dot{A}_{\mathrm{T}}(t)
$$

Once $N_{T}\left(\underline{x}, t^{n+1}\right)$ is known solving 19 , the PDF equation 16 is advanced in time with nucleation and agglomeration:

$$
\begin{aligned}
\bar{P}\left(v^{*} ; t^{n+1}\right) & =\alpha_{H_{o}} \delta\left(v_{o}-v^{*}\right)+\alpha_{A_{i}} \delta\left(v_{i}-v^{*}\right) \\
& +\left(1-\alpha_{H_{o}}-\alpha_{A_{T}}\right) \bar{P}\left(v^{*} ; t^{n+\frac{1}{2}}\right)
\end{aligned}
$$

with $\alpha_{H_{o}}$ the relative increase of $\bar{P}\left(v_{o} ; t^{n+\frac{1}{2}}\right)$ by nucleation (and decrease of $\bar{P}\left(v^{*} ; t^{n+\frac{1}{2}}\right)$ for $\left.v^{*} \neq v_{o}\right), \alpha_{A_{i}}$ the relative increase/decrease due to agglomer- 
ation and $\alpha_{A_{T}}$ the total agglomeration sink defined by Eq. (16):

$$
\begin{aligned}
\alpha_{H_{o}} & =\frac{\dot{H}_{o}\left(t^{n+\frac{1}{2}}\right)}{N_{\mathrm{T}}\left(t^{n+1}\right)} \cdot \delta t, \\
\alpha_{A_{i}} & =\frac{\dot{A}_{i}\left(t^{n+\frac{1}{2}}\right)}{N_{T}\left(t^{n+1}\right)} \cdot \delta t, \\
\alpha_{A_{T}} & =\frac{\dot{A}_{T}\left(t^{n+\frac{1}{2}}\right)}{N_{T}\left(t^{n+1}\right)} \cdot \delta t .
\end{aligned}
$$

According to Eq. (17), integrating over $I_{v_{i}}$ and multiplying by $N_{P}$ the PDF evolution given by the relation (20) leads to the evolution of the number of stochastic particles per section. This discretised time evolution is organised as:

$$
n_{P_{i}}\left(t^{n+1}\right)=n_{P_{i}}\left(t^{n+\frac{1}{2}}\right)+\Delta n_{P_{i}}\left(t^{n+\frac{1}{2}}\right),
$$

with increments $\Delta n_{P_{i}}\left(t^{n+\frac{1}{2}}\right)$ in the form of real numbers, which will need to 205 be transformed subsequently into integer numbers of particles in the Monte Carlo algorithm. From 20,

$$
\begin{aligned}
\Delta n_{P_{o}}\left(t^{n+\frac{1}{2}}\right) & =\left(\alpha_{H_{o}}+\alpha_{A_{o}}\right) N_{P}-\left(\alpha_{H_{o}}+\alpha_{A_{T}}\right) n_{P_{o}}\left(t^{n+\frac{1}{2}}\right) \\
& +\alpha_{R_{o}} N_{P}, \\
\Delta n_{P_{i}}\left(t^{n+\frac{1}{2}}\right) & =\alpha_{A_{i}} N_{P}-\left(\alpha_{H_{o}}+\alpha_{A_{T}}\right) n_{P_{i}}\left(t^{n+\frac{1}{2}}\right) \\
& +\alpha_{R_{i}} N_{P} \text { for } i \neq o .
\end{aligned}
$$

The terms proportional to $\alpha_{R_{i}}$ are the accumulation of the round-off error, which goes to zero for $N_{P} \rightarrow \infty$. Cumulated over the iterations, this will impact on particles when $\alpha_{R_{i}} \geq 1 / N_{P}$. At every iteration, $\Delta n_{P_{i}}\left(t^{n+\frac{1}{2}}\right)$ is thus decomposed into its integer and fractional (or decimal) parts. The fractional part $\left\{\Delta n_{P_{i}}\left(t^{n+\frac{1}{2}}\right)\right\}$ is defined from the nearest integer $\left[\Delta n_{P_{i}}\left(t^{n+\frac{1}{2}}\right)\right\rceil$,

$$
\left\{\Delta n_{P_{i}}\left(t^{n+\frac{1}{2}}\right)\right\}=\Delta n_{P_{i}}\left(t^{n+\frac{1}{2}}\right)-\left\lfloor\Delta n_{P_{i}}\left(t^{n+\frac{1}{2}}\right)\right\rceil .
$$

The integer part $\left\lfloor\Delta n_{P_{i}}\left(t^{n+\frac{1}{2}}\right)\right\rceil$ sets the variation of the number of stochastic particles within a section during the reallocation step corresponding to nucleation and agglomeration. The following Monte Carlo algorithm is applied:

- If $\left\lfloor\Delta n_{P_{i}}\left(t^{n+\frac{1}{2}}\right)\right\rceil$ is negative, a random number - $\left\lfloor\Delta n_{P_{i}}\left(t^{n+\frac{1}{2}}\right)\right\rceil$ of stochastic particles is picked among the $n_{P_{i}}\left(t^{n+\frac{1}{2}}\right)$ present in $I_{v_{i}}$. 
- All the picked particles from all $I_{v_{i}}$ intervals $(i=1, \cdots, M)$ constitute an ensemble $\mathcal{P}\left(t^{n+\frac{1}{2}}\right)$ of particles whose characteristic size needs to change.

- If $\left\lfloor\Delta n_{P_{i}}\left(t^{n+\frac{1}{2}}\right)\right\rceil$ is positive, $\left\lfloor\Delta n_{P_{i}}\left(t^{n+\frac{1}{2}}\right)\right\rceil$ particles are taken from $\mathcal{P}\left(t^{n+\frac{1}{2}}\right)$ and allocated to $I_{v_{i}}$ at the representative size $v_{i}^{\star}\left(t^{n+\frac{1}{2}}\right)$, defined to conserve mass, as discussed in the next subsection.

The larger the total number of stochastic particles $N_{P}$, the smaller the relative contribution of the decimal part $\left\{\Delta n_{P_{i}}\left(t^{n+\frac{1}{2}}\right)\right\}$ to $\Delta n_{P_{i}}\left(t^{n+\frac{1}{2}}\right)$. This residual decimal part defines $N_{i}^{R}\left(t^{n}\right)$, a residual number density of physical particles in the section $v_{i}$, which is computed at time $t^{n}$ following (17)

$$
N_{i}^{R}\left(t^{n}\right)=\frac{\left\{\Delta n_{P_{i}}\left(t^{n-\frac{1}{2}}\right)\right\}}{N_{P}} N_{T}\left(t^{n}\right),
$$

where $\Delta n_{P_{i}}\left(t^{n-\frac{1}{2}}\right)$ denotes $\Delta n_{P_{i}}$ of the previous iteration in time. The growth/loss of the physical particles represented by this number density residual $N_{i}^{R}\left(t^{n}\right)$ is not included in the stochastic particles and needs a separate solving, between $t^{n}$ and $t^{n+\frac{1}{2}}$ (i.e., simultaneously with growth/loss for the stochastic particles Eq. (18)) This is done with a sectional method based on the 3-point discretisation for particle growth/loss (Park \& Rogak, 2004) Appendix A. Then, $N_{i}^{R}\left(t^{n+\frac{1}{2}}\right)$ is known and $\alpha_{R_{i}}$ is obtained from

$$
\alpha_{R_{i}}=\frac{N_{i}^{R}\left(t^{n+\frac{1}{2}}\right)}{N_{T}\left(t^{n+1}\right)},
$$

and applied to Eqs. 25) and (26) to compute $\Delta n_{P_{i}}\left(t^{n+\frac{1}{2}}\right)$. For sufficiently large values of $N_{P}$, typically $10^{5}$ as shown thereafter, the residual number density of particles is expected to be negligible and will not perturb much the accuracy of the method. Then, $\alpha_{R}\left(v_{i}\right)$ can be set to zero in the relations (25) and (26). However as shown below, accounting for the contribution of the residual part allows for reducing $N_{P}$ (such as $10^{3}$ or less) and therefore the CPU time.

Optionally, a trigger can also be set so that when the number of stochastic particles present in a given section becomes too small, the surface growth/loss is then fully solved through the evolution of $N_{i}^{R}(t)$. In practice, a trigger of 5 particles per section is used and has been found to be sufficient to avoid any noise on the tails of distributions. 
Table 1: Growth parameters

\begin{tabular}{lll}
\hline Case & $1(\mathrm{a})$ & $1(\mathrm{~b})$ \\
\hline Initial & 1 for $0.2 \leq v ; 0$ else & $\delta(1)$ \\
Growth kernel & 0.05 & $v$ \\
Agglo. kernel & 0 & 0 \\
Number of sections & 20 & 40 \\
Grid type & unif. $\Delta v=0.2$ & geo. $F_{s}=2$ \\
Size range & $0-4$ & $0.7-7.3 \cdot 10^{11}$ \\
\hline
\end{tabular}

Figure 1 displays a flowchart summarising the method.

\subsubsection{Agglomeration source}

The method proposed in Kumar \& Ramkrishna (1996a) is retained for computing the agglomeration source $A_{i}(t)$ of Eq. (22). For any colliding particles of volume $v$ in section $i$ and $\bar{v}$ in section $j$, the collision kernel $\beta(v, \bar{v})$ is assumed fixed to $\beta\left(v_{i}, v_{j}\right)=\beta_{i, j}$. Particles formed by agglomeration are distributed in the sections in a manner that conserves the zeroth and first moments of the PSD, namely number and mass. This method avoids the evaluation of the double integrals of the collision kernel and is therefore computationally efficient (see Kumar \& Ramkrishna (1996a) for more details).

The agglomeration source used in $(22)$ reads

$$
\begin{aligned}
\dot{A}_{i}(t) & =\sum_{\substack{j, k \\
v_{i-1} \leq v_{j}+v_{k} \leq v_{i+1}}}^{k \leq j \leq i}\left(1-\frac{\delta_{j, k}}{2}\right) \eta \beta_{j, k} N_{j}(t) N_{k}(t) \\
& -N_{i}(t) \sum_{k=1}^{M} \beta_{i, k} N_{k}(t),
\end{aligned}
$$

with

$$
\eta= \begin{cases}\frac{v_{i+1}^{\star}-\left(v_{j}^{\star}+v_{k}^{\star}\right)}{v_{i+1}^{\star}-v_{i}^{\star}} & \text { if } v_{i}^{\star} \leq v_{j}^{\star}+v_{k}^{\star} \leq v_{i+1}^{\star}, \\ \frac{v_{i-1}^{\star}-\left(v_{j}^{\star}+v_{k}^{\star}\right)}{v_{i-1}^{\star}-v_{i}^{\star}} & \text { if } v_{i-1}^{\star} \leq v_{j}^{\star}+v_{k}^{\star} \leq v_{i}^{\star},\end{cases}
$$

In the hybrid stochastic/fixed-sectional approach, the characteristic volume $v_{i}^{\star}$ must be representative of the average mass contained in the $i$-th section. $v_{i}^{\star}$ is calculated dynamically, depending on the volumes of the stochastic particles contained in both the section and the residual terms resulting from 
the roundoff,

$$
v_{i}^{\star}\left(t^{n+\frac{1}{2}}\right)=\frac{\left(N_{T}\left(t^{n}\right) / N_{P}\right) \sum_{k=1}^{n_{P_{i}}(t)} v_{i}^{k}\left(t^{n+\frac{1}{2}}\right)+N_{i}^{R}\left(t^{n+\frac{1}{2}}\right) v_{i}^{\star}\left(t^{n}\right)}{\left(N_{T}\left(t^{n}\right) / N_{P}\right) n_{P_{i}}\left(t^{n+\frac{1}{2}}\right)+N_{i}^{R}\left(t^{n+\frac{1}{2}}\right)},
$$

where $v_{i}^{k}=v^{k}$ if $v^{k} \in I_{v_{i}}$ and $v_{i}^{k}=0$ otherwise, $n_{P_{i}}\left(t^{n+\frac{1}{2}}\right)$ is the number of stochastic particles in the $i$-th section (Eq. (17)) and $N_{i}^{R}\left(t^{n+\frac{1}{2}}\right)$ is the residual number density of the particles in the section after applying surface gross or loss. $v_{i}^{\star}$ needs to be updated again after reallocation of the particles due to agglomeration, to provide $v_{i}^{\star}\left(t^{n+1}\right)$ from $(32)$ with $N_{T}\left(t^{n+1}\right), v_{i}^{k}\left(t^{n+1}\right)$, $N_{i}^{R}\left(t^{n+1}\right), v_{i}^{\star}\left(t^{n+\frac{1}{2}}\right), n_{P_{i}}\left(t^{n+1}\right)$.

Once $v_{i}^{\star}$ determined, the particles reassigned in the $i$-th section are distributed in this section following a two-step process:

- First, the $\left\lfloor\Delta n_{P_{i}}\left(t^{n+\frac{1}{2}}\right)\right\rceil$ particles are allocated randomly within the section at sizes $v^{k}\left(t^{n+\frac{3}{4}}\right)$, which are samples of a random variable $v$ following a target piecewise linear distribution defined by the probability density function,

$$
p\left(v \mid v_{i}^{\text {inf }}, v_{i}^{\text {sup }}, w_{i}, w_{i+1}\right)=2 \frac{w_{i}\left(v_{i}^{\text {sup }}-v\right)+w_{i+1}\left(v-v_{i}^{\text {inf }}\right)}{\left(w_{i}+w_{i+1}\right) \Delta v_{i}^{2}} .
$$

In this distribution, the weights, $w_{i}$, are calculated from the variations of the number densities at $v_{i}^{\star}$,

$$
\begin{aligned}
w_{i} & =\Delta n_{i}\left(t^{n+\frac{1}{2}}\right)+\frac{\Delta n_{i}\left(t^{n+\frac{1}{2}}\right)-\Delta n_{i-1}\left(t^{n+\frac{1}{2}}\right)}{v_{i}^{\star}-v_{i-1}^{\star}}\left(v_{i}^{i n f}-v_{i-1}^{\star}\right), \\
w_{i+1} & =\Delta n_{i}\left(t^{n+\frac{1}{2}}\right)+\frac{\Delta n_{i+1}\left(t^{n+\frac{1}{2}}\right)-\Delta n_{i}\left(t^{n+\frac{1}{2}}\right)}{v_{i+1}^{\star}-v_{i}^{\star}}\left(v_{i}^{\text {sup }}-v_{i}^{\star}\right),
\end{aligned}
$$

with $\Delta n_{i}(t)=\Delta n_{P_{i}}(t) N_{T}(t) /\left(N_{P} \Delta v_{i}\right)$ (Eq. (17)). Such random piecewise linear distribution secures a continuous distribution of the stochastic particles. However, it does not guarantee strict volume/mass conservation by itself.

- Mass conservation is achieved in a second step by calculating a correc- 
Table 2: Agglomeration parameters

\begin{tabular}{lll}
\hline Case & $2(\mathrm{a})$ & $2(\mathrm{~b})$ \\
\hline Initial & $e^{-v}$ & $e^{-v}$ \\
Agglo. kernel & 1 & $v_{i}+v_{j}$ \\
Number of sections & 40 & 40 \\
Grid type: Exponential, $\alpha$ & 1.17 & 1.25 \\
Size range & $6.7 \cdot 10^{-2}-209$ & $6.7 \cdot 10^{-2}-2006$ \\
\hline
\end{tabular}

tive factor $K_{i}$

$$
K_{i}=\frac{v_{i}^{\star}\left(t^{n+\frac{1}{2}}\right)}{\left(1 / n_{P_{i}}\left(t^{n+\frac{3}{4}}\right)\right) \sum_{k=1}^{n_{P_{i}}\left(t^{n+1}\right)} v_{i}^{k}\left(t^{n+\frac{3}{4}}\right)},
$$

then,

$$
v_{i}^{k}\left(t^{n+1}\right)=K_{i} v_{i}^{k}\left(t^{n+\frac{3}{4}}\right),
$$

\section{both stochastic and sectional parts.}

The characteristic time step size of the nucleation-agglomeration sub-step of the algorithm is determined to limit the relative change of the distribution 


$$
\delta t_{A}=(\gamma+\sigma) \frac{N_{T}}{\left|\dot{H}_{o}+\dot{A}_{T}\right|+\sum_{i=1}^{M}\left|\dot{A}_{i}\right|},
$$

with $\sigma=0.02$ in the simulations presented thereafter. If particle nucleation dominates, as in the beginning of a calculation with a negligible initial distribution mostly present in the smallest section with also very few exchange of particles between sections, larger time steps may be allowed to let $N_{T}$ increase faster until the exchange of particles between sections becomes significant, then $\gamma=1$ is used in (37). This specific 'nucleation dominated' regime is considered reached at a given time in a simulation if

$$
\begin{aligned}
\left|\dot{H}_{o}(t)+\dot{A}_{T}(t)\right| & >100 \cdot \sum_{i=1}^{M}\left|\dot{A}_{i}(t)\right|, \\
N_{0}(t) / N_{T}(t) & >0.99 .
\end{aligned}
$$

Otherwise, $\gamma=0$ is imposed in (37) to solve for the more general regimes of PSD evolution.

For the test cases considered in this work, $\delta t_{G} \leq \delta t_{A}$ and one or several surface growth/loss sub-iterations can be applied between two agglomeration/nucleation sub-iterations. $\delta t_{G}$ is then further adjusted so that $\delta t_{A}$ is one of its multiple, still verifying the stability condition.

\section{Canonical test cases}

Four main representative cases for which analytical solutions exist are considered. Sectional methods, based on two discretisations of the growth term, and the hybrid stochastic/sectional approach discussed above are applied to simulate these canonical problems.

The number of sections set to discretise the normalised problems is fixed to 20,40 or 80 depending on the case, for various size ranges (see the details in Tables 1 to 4 . Following the literature, three types of grid discretisation are used: uniform, geometric and exponential. Defining $v_{i}^{\text {inf }}$ the inferior boundary of section i, the uniform grid reads

$$
v_{i}^{i n f}=v_{0}^{i n f}+i \Delta v
$$


Table 3: Growth/Loss \& agglomeration parameters

\begin{tabular}{llllll}
\hline Case & $3(\mathrm{a})$ & $3(\mathrm{~b})$ & $3(\mathrm{c})$ & $3(\mathrm{~d})$ & $3(\mathrm{e})$ \\
\hline Initial & $e^{-v}$ & $e^{-v}$ & $e^{-v}$ & $e^{-v}$ & $e^{-v}$ \\
Growth kernel & $v$ & $v$ & $v$ & $v$ & $-v$ \\
Agglo. kernel & 0.1 & 1 & 10 & 1 & 1 \\
Number of sections & 40 & 40 & 40 & 80 & 40 \\
Geometric grid, $F_{s}$ & 2 & 2 & 2 & $\sqrt{2}$ & 2 \\
Size range & $6.7 \cdot 10^{-4}-7.3 \cdot 10^{8}$ & $8.3 \cdot 10^{-4}-9.1 \cdot 10^{8}$ & $6.7 \cdot 10^{-11}-73$ \\
\hline
\end{tabular}

the geometric grid is constructed as in Park \& Rogak (2004) following

$$
v_{i}^{\text {inf }}=v_{0}^{\text {inf }} F_{s}^{i}
$$

and the exponential grid as in Rigopoulos \& Jones $(2003){ }^{2}$

$$
v_{i}^{\text {inf }}=v_{0}^{\text {inf }}+v_{0}^{\text {inf }} \frac{1-\alpha^{i}}{1-\alpha} .
$$

The values of $F_{s}$ and $\alpha$ are given in Tables 1 to 4 .

The first main case features only growth and is broken into two subcases 1(a) and 1(b). Following Sewerin \& Rigopoulos (2017), case 1(a) of Table 1 considers the advection of a unit step distribution, whose exact solution is a pure advection of the step function at the constant normalised speed $G=0.05$.

${ }_{290}$ Case 1 (b) in Table 1 is from Park \& Rogak (2004) and represents the pure growth of a set of mono-disperse particles. The initial particle size distribution is a delta function, which is translated in size space at a speed proportional to the particle volume $G(v)=v$. Cases $1(\mathrm{a})$ and $1(\mathrm{~b})$ are quite stringent, since numerical diffusion can transform the expected delta function into a poly-disperse distribution.

The second case in Table 2 is pure agglomeration. Case 2(a) is with a fixed agglomeration frequency, as proposed in Rigopoulos \& Jones (2003). From an initial exponential distribution $\exp (-v)$, the analytical solution of

\footnotetext{
${ }^{2}$ Exponent $i$ is used instead of $i-1$ of Rigopoulos \& Jones (2003) as $i$ begins at 0 in the present case.
} 
the time evolution of the PSD was discussed in Scott (1968),

$$
n(v ; t)=\frac{4}{(t+2)^{2}} \exp \left(-\frac{2 v}{t+2}\right) .
$$

Case 2(b) is with a non-uniform collision frequency. Here, the Golovin sum kernel $\beta\left(v_{i}, v_{j}\right)=\left(v_{i}+v_{j}\right)$ is retained to mimic the expected increase with volume of the collision frequency between two particles of characteristic sizes $v_{i}$ and $v_{j}$. Starting from the same initial exponential distribution, the analytical solution of the PSD reads (Scott, 1968; Rigopoulos \& Jones, 2003)

$$
n(v ; t)=\left(\frac{1-\theta}{\theta^{1 / 2}}\right) \cdot \frac{\exp (-v(\theta+1))}{v} \cdot I_{1}\left[2 v \theta^{1 / 2}\right]
$$

where $\theta=1-\exp (-t)$ and $I_{1}$ denotes the first order Bessel-I function.

In a third series of cases, agglomeration with either surface growth or loss is considered (Table 3). Starting from an initial exponential distribution, the time evolution follows (Ramabhadran et al., 1976):

$$
n(v ; t)=\frac{4}{\left(2+\beta_{0} t\right)^{2}} \exp \left(-\frac{2 v \exp (-t)}{2+\beta_{0} t}-t\right) .
$$

The value of the size-independent collision kernel $\beta_{o}$ is varied by two orders of magnitude $\left(\beta_{o}=0.1\right.$ case $3(\mathrm{a}), \beta_{o}=1$ case $3(\mathrm{~b}), 3(\mathrm{~d})$ and $3(\mathrm{e}), \beta_{o}=10$ case $3(\mathrm{c})$, Table 3). In case $3(\mathrm{~d})$, in the comparison between the hybrid method and the fixed-sectional one, the latter benefits from twice the number of sections. In case 3(e), surface loss is applied instead of surface growth with $G(v)=-v$. The initial distribution and collision kernel are the same as in case $3(\mathrm{~b})$.

Case 4 is the evolution of an initial exponential distribution submitted to nucleation and growth (Table 4), evolving into a uniform distribution for large times. The normalised nucleation kernel $\dot{H}\left(v_{o}, t\right)$ is fixed to unity in this last test case.

\section{Results}

Analytical solutions are compared with simulation results. Simulations were run for all cases of Tables 1 to 4 with the hybrid stochastic/fixedsectional approach and with the standard fixed-sectional method. Convection in size space was solved using either a 2-point or a 3-point algorithm Park 
Table 4: Nucleation \& growth parameters

\begin{tabular}{ll}
\hline Case & 4 \\
\hline Initial & $10^{-5} \delta\left(v_{o}\right)$ \\
Growth kernel & $v$ \\
Number of sections & 40 \\
Geometric grid, $F_{s}$ & 2 \\
Size range & $0.7-7.3 \cdot 10^{11}$ \\
\hline
\end{tabular}

\& Rogak, 2004) (see also Appendix A). Only test case 1(a) was not run with the 3-point algorithm, which is not designed to solve for growth on a uniform grid.

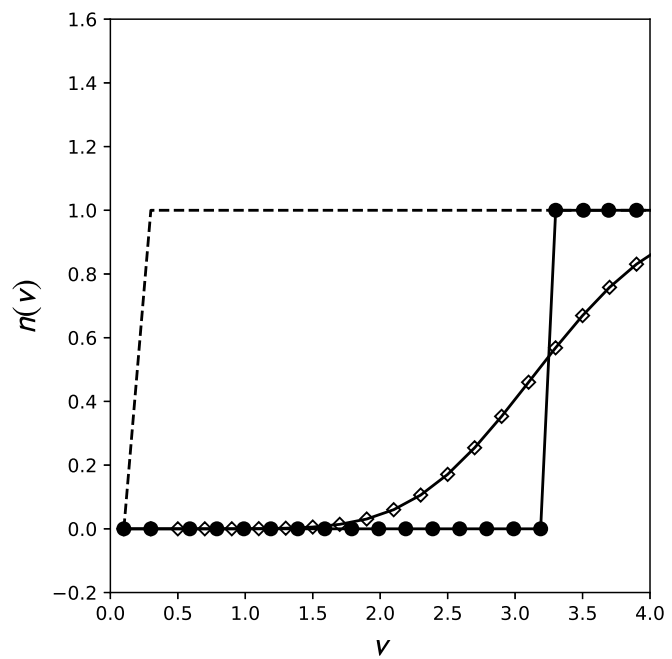

Figure 2: Particle Size Distribution $n(v ; t)$. Growth: case 1(a) of Table 1. Dashed line: initial distribution (jump is between centred sections values). Normalised time $t=60$. Line with empty diamonds: 2-point sectional method. Line with full circles: hybrid method, $N_{P}=10^{3}$ (values are shown at $v_{i}^{\star}$ (Eq. (32)).

In case $1(\mathrm{a})$, the hybrid method perfectly reproduces the analytical solution (Fig. 2), with the pure convection of the step function. As expected, applying growth directly on the stochastic particles enables to convect the distribution in size space with no numerical diffusion. Similar results were obtained by Sewerin \& Rigopoulos (2017) using explicit adaptive grid method (EAGM), while in Fig. 2, the 2-point fixed sectional approach yields results close to those of fully upwinded orthogonal collocation finite element method (OCFEM), see Fig. 7 of Rigopoulos \& Jones (2003). 


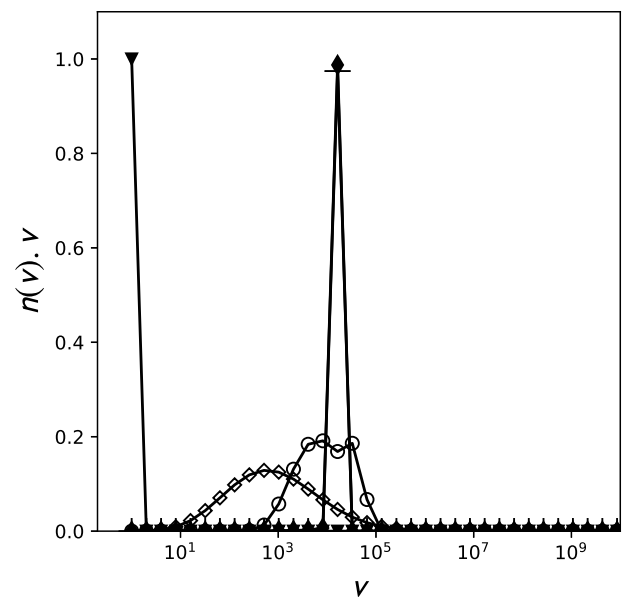

Figure 3: Size Distribution $n(v ; t) \cdot v$. Growth: case 1(b) of Table 1. Triangle: Initial distribution. Plus symbol: Analytical solution. Line with diamonds: sectional 2-point method. Line with circles: sectional park 3-point method (Park \& Rogak, 2004). Diamond symbols: hybrid method without residual term, $\alpha_{R}=0, N_{P}=10^{3}$.

Case 1(b) of Table 1 features an initial monodisperse distribution submitted to growth only, with a particle surface growth rate proportional to the particle volume. The expected solution is thus a translation of the distribution in size space. Figure 3 shows that the fixed-sectional methods (line with diamonds and circles) would need much more advanced numerics to capture this extreme case. However, the hybrid method operating here without any residual, returns the exact solution. In specific aerosol flow zones, where nucleation and pure growth can dominate the physics, the spurious spreading of the distributions observed with sectional methods could strongly impair the calibration of the physical models.

The pure agglomeration case 2(a) of Table 2, with a fixed agglomeration frequency, is simulated with the hybrid approach for $N_{P}=10^{3}, 10^{4}$ and $10^{5}$. The initial distribution and the solutions at two successive times are shown in Fig. 4. To assess the impact of the residual number density (Eq.(28)), Figs 4(a), 4(b) and 4(c) are obtained forcing $\alpha_{R}=0$ (Eq. (29)). As $N_{P}$ is decreased from $10^{5}$ to $10^{3}$, the effect of the roundoff then becomes visible. The introduction of the procedure discussed above to deal with the residual part, allows for alleviating this effect to better match the solution (Fig. 4(d)) The case 2(b) of Table 2 with the Golovin agglomeration kernel (Eq. (44)) 


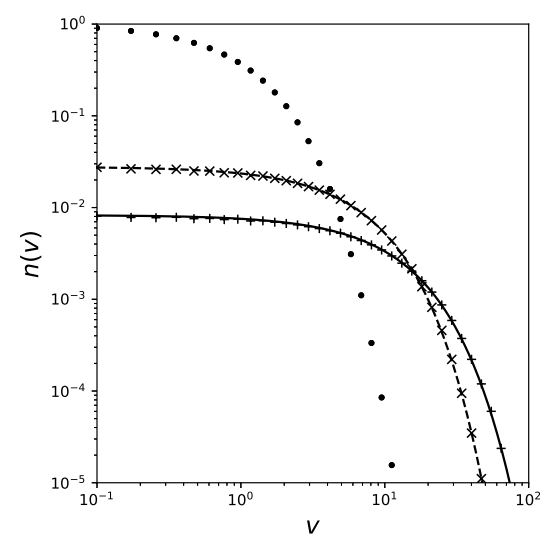

(a) $N_{\mathrm{P}}=10^{5}, \alpha_{R}=0$

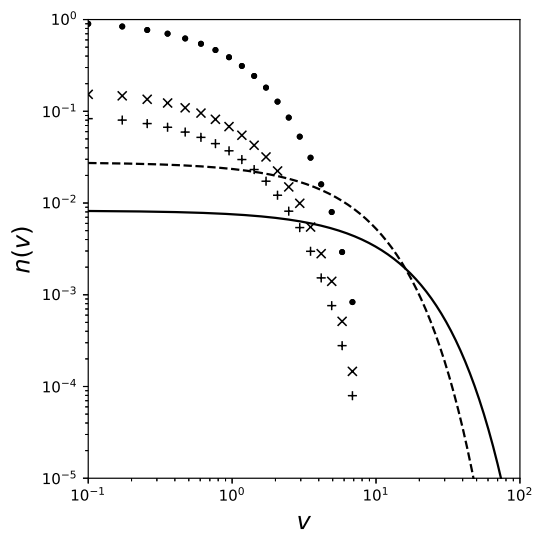

(c) $N_{\mathrm{P}}=10^{3}, \alpha_{R}=0$

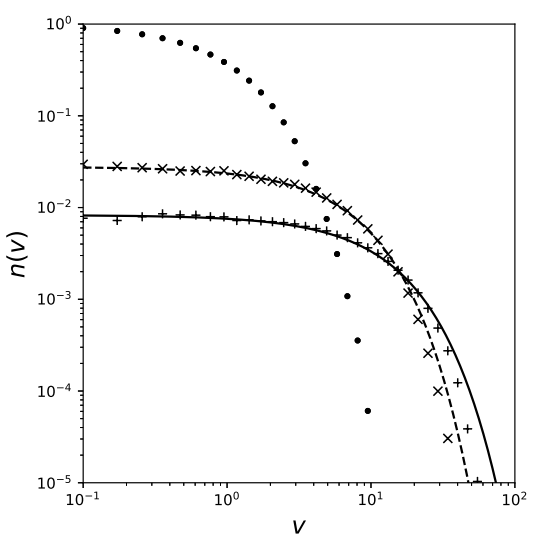

(b) $N_{\mathrm{P}}=10^{4}, \alpha_{R}=0$

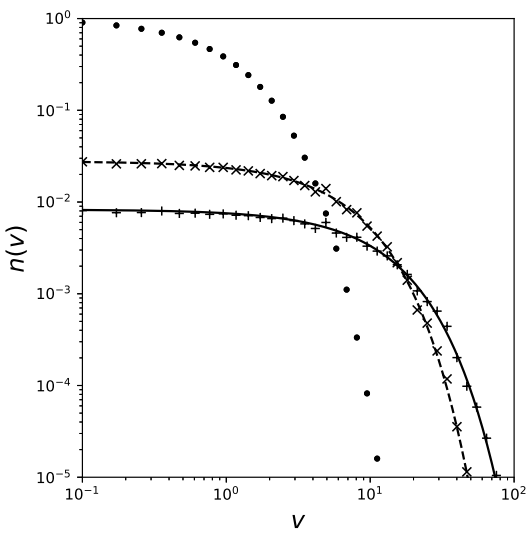

(d) $N_{\mathrm{P}}=10^{3}, \alpha_{R}$ by 29 .

Figure 4: Particle Size Distribution $n(v ; t)$. Size independent agglomeration: case 2(a) of Table 2 (Eq. (43)). Points: initial distribution. $t=10$, dashed line: analytical solution, crosses: hybrid method. $t=20$, solid line: analytical solution, plus: hybrid method. (a)-(c): without residual term, $\alpha_{R}=0$. (d): with residual term (Eq. 29p).

is also perfectly reproduced (Fig. 5).

The cases 3(a), (b) and (c), with both surface growth and agglomeration of the particles, are shown in Fig. 6. Here comparisons are made between the exact solution given by the relation (45), the solution with the hybrid method and the solutions with the fixed-sectional method using the classical 2-point and 3-point discretisation for growth (Park \& Rogak, 2004). Three collision parameters are applied $\left(\beta_{o}=0.1,1.0\right.$ and 10) and results are given 


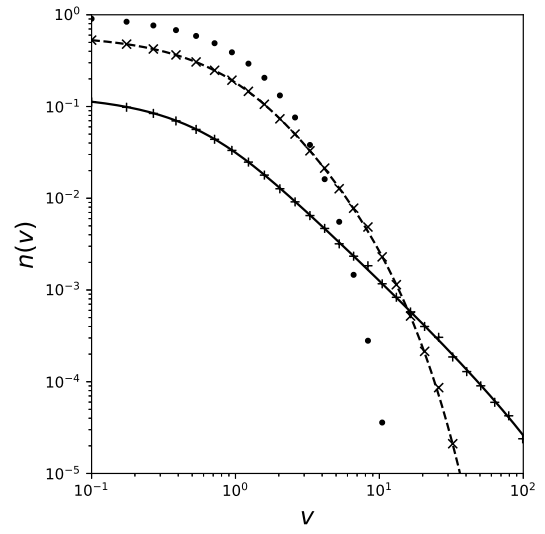

Figure 5: Particle Size Distribution $n(v ; t)$. Size dependant agglomeration: case 2(b) of Table 2 (Eq. (43)). Points: initial distribution. $t=0.5$, dashed line: analytical solution, crosses: Hybrid method. $t=2$, solid line: analytical solution, plus: hybrid method, $N_{P}=10^{4}$.

in both linear and log scale for the amplitude of the PSD. As expected, the sectional 3-point method is more accurate than its 2-point counterpart (Fig. 6). The hybrid stochastic/fixed-sectional method returns the best results, with a better prediction of the peak value and less spurious diffusion of the distribution. The comparison between log-scale and linear plots also illustrates the risk in concluding from log-plots only, specifically for physical problems with high sensitivity to small fluctuations of size around a targeted peak level. Even in case $3(\mathrm{~d}), \beta_{o}=1$ with a much finer grid for the sectional method, which results in a number of sections multiplied by two $(M=80)$, still keeping $(M=40)$ for the hybrid method, the latter achieves a similar level of accuracy as compared with the three-point fixed-sectional method and performs better than the two-point one (Fig. 7). The case 3(e) with surface loss (Fig. 8) combined with agglomeration shows that the sectional 3 -point method over-estimates the peak value of the distribution with a too narrow size distribution, as a result of the effect of the flux-limiting in case of negative velocity. On the opposite, the hybrid method follows with much better accuracy the analytical solution.

In case 4 of Table 4, simultaneous growth and constant nucleation are applied as in Park \& Rogak (2004). A negligible initial distribution, orders of magnitude smaller than the expected converged solution, is introduced to 


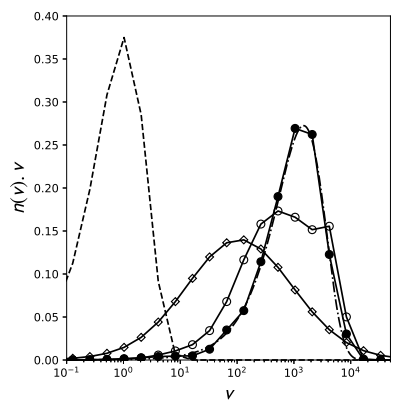

(a) $\beta_{o}=0.1$ case $3(\mathrm{a})$

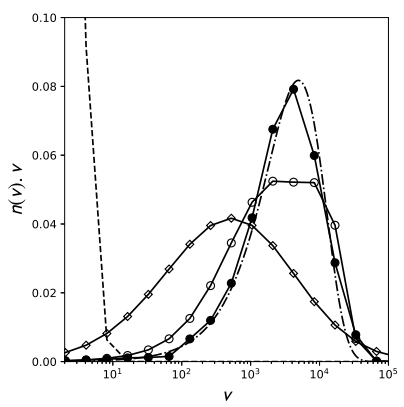

(c) $\beta_{o}=1.0$ case $3(\mathrm{~b})$

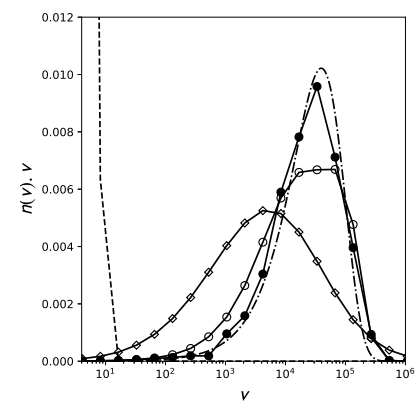

(e) $\beta_{o}=10.0$ case $3(\mathrm{c})$

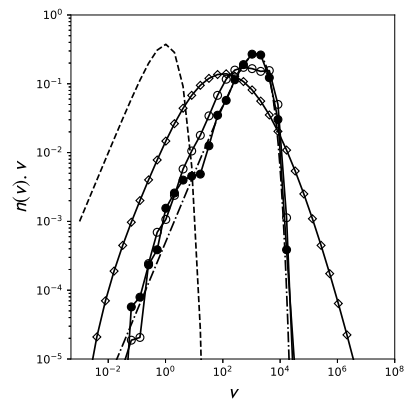

(b) log-scale

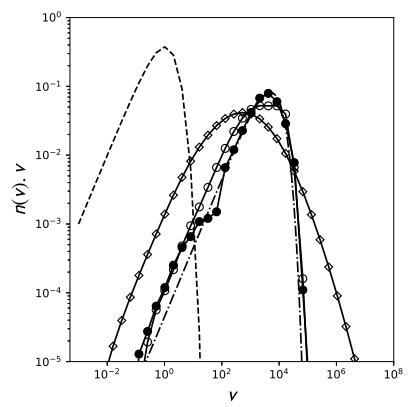

(d) log-scale

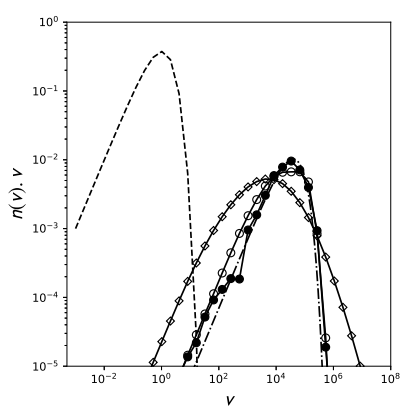

(f) $\log$-scale

Figure 6: Size Distribution $n(v ; t) \cdot v$. Surface growth $(G(v)=v)$ \& agglomeration: case 3(a), 3(b), 3(c) of Table 3 $\beta_{o}$ : size-independent collision kernel (Eq. (45)). Dashed line: initial distribution. $t=7$, dashed dotted line: analytical solution. Line with empty diamonds: 2-point sectional method. Line with empty circles: 3-point sectional method. Line with full circles: hybrid method, $N_{P}=10^{3}$. 


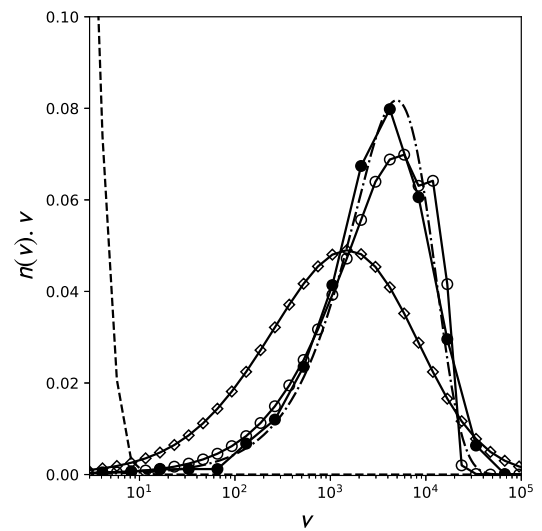

Figure 7: Size Distribution $n(v ; t) \cdot v$. Growth \& agglomeration: case 3(d) of Table 3 Dashed line: initial distribution. Dashed dotted line: analytical solution at $t=7$. Line with empty diamonds: 2-point sectional method ( $M=80$ sections). Line with empty circles: 3-point sectional method ( $M=80$ sections). Line with full circles: hybrid method, $N_{P}=10^{3}$ ( $M=40$ sections $)$.

are applied and results are seen in Fig. 9. The hybrid method reproduces the steep moving front induced by simultaneous growth and nucleation, with results closer to the analytical number density than those of the 3-point fixedsectional method over the whole range of particles sizes. The 3-point fixedsectional method overestimates the number density for $v \in\left[2,10^{3}\right]$. Then, from sizes around $2 \cdot 10^{3}$ up to the theoretically moving front, the number density is largely underestimated by the 3-point fixed-sectional method. The 2-point method is highly diffusive as compared with the 3-point and hybrid approaches.

380 In addition to the visualisation of the PSD distributions, to verify that no additional error accumulates with the hybrid method compared with the sectional ones, three measures of the error against $n(v ; t)$, the exact solutions of the canonical test cases given by (43), (44) and (45), have been computed. $\epsilon_{M_{q}}$, with $q=1$ or 2 , is the departure in $\%$ between the $q$-th moment of the 385 analytical solution

$$
M_{q}(t)=\int_{v_{o}}^{v_{M}} n(v ; t) v^{q} d v,
$$




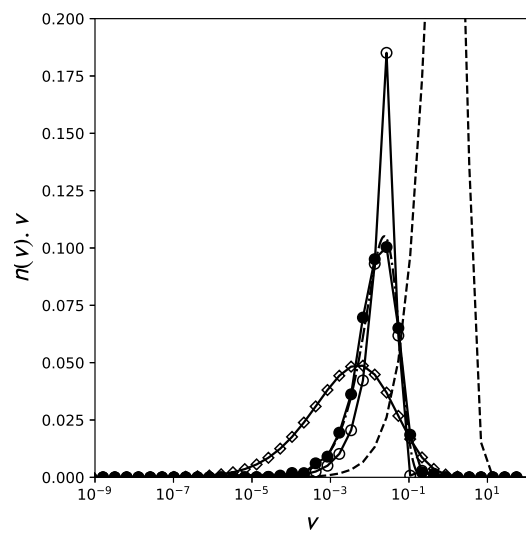

(a) $\beta_{o}=1.0$ case $3(\mathrm{e})$

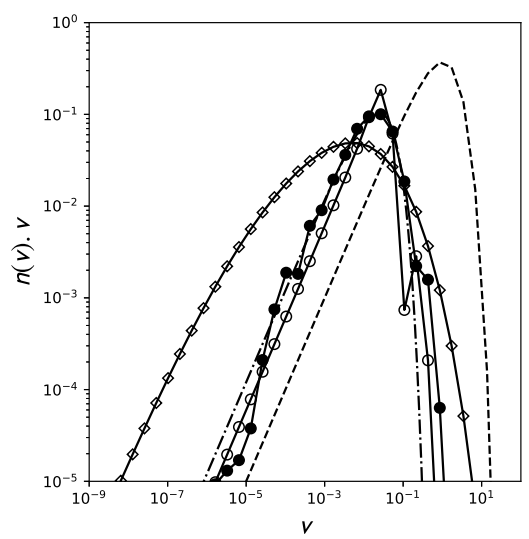

(b) log-scale

Figure 8: Size Distribution $n(v ; t) \cdot v$. Surface loss $(G(v)=-v)$ \& agglomeration: case 3(e) of Table $3 . \quad \beta_{o}$ : size-independent collision kernel (Eq. (450). Dashed line: initial distribution. $t=5$, dashed dotted line: analytical solution. Line with empty diamonds: 2-point sectional method. Line with empty circles: 3-point sectional method. Line with full circles: hybrid method, $N_{P}=10^{3}$.

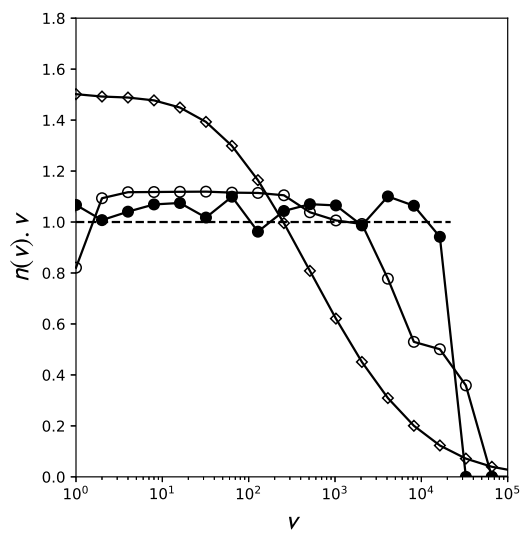

(a) case 4

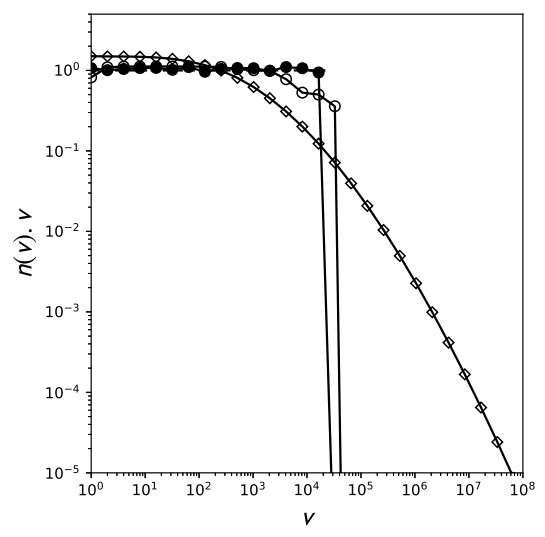

(b) log-scale

Figure 9: Size Distribution $n(v ; t) \cdot v$. Nucleation and growth Case 4 of Table 4 . Dash-dot line: analytical solution at $t=9.7$. Line with empty diamonds: 2-point sectional method. Line with empty circles: 3-point sectional method. Line with full circles: hybrid method, $N_{P}=10^{3}$. 
against either the moment of the hybrid method

$$
M_{q}^{H y b}(t)=\sum_{i=1}^{M}\left(\frac{N_{T}(t)}{N_{P}} \sum_{k=1}^{n_{P_{i}}(t)}\left(v_{i}^{k}(t)\right)^{q}+N_{i}^{R}(t)\left(v_{i}^{\star}(t)\right)^{q}\right)
$$

with $v_{i}^{\star}(t)$ from 32 , or the moment of the fixed-sectional method

$$
M_{q}^{S e c}(t)=\sum_{i=1}^{M} N_{i}(t) v_{i}^{q}
$$

As expected, the fixed-sectional methods are accurate on the integral first moment of the distribution (Table 5). The hybrid stochastic/fixed-sectional approach returns values of $\epsilon_{M_{1}}$ which are also of the order of a few percent. The error on the second moment, $\epsilon_{M_{2}}$, reveals the accumulation of a much larger level of error with the sectional methods as soon as surface growth or loss is acting on the PSD (Table 5), confirming the better description of PSD shape with the hybrid approach.

The third measure is based on the Earth Mover's Distance (EMD) (Rubner et al., 1998), or Wasserstein metric, computed using the package 'emdist' of the cran project (Urbanek, 2012). EMD is a statistical metric that represents the distance between two probability distributions. It does not require the same discretization of the compared distributions. EMD has previously been used for Particle Size Distributions and compared with other distance measures ( $\mathrm{Hu}$ et al., 2018). The EMD is normalised by the standard deviation of the exact distribution. The hybrid method reports for $\epsilon_{E M D}$ similar trends than with the fixed-sectional methods (Table 5), with better estimation in case 3 (agglomeration and growth) and case 4 (nucleation and growth), as expected from the above discussion.

With fixed-sectional methods, the error on the PSD usually increases as $\mathcal{R}_{\tau}$, the ratio between the characteristic growth and agglomeration times, decreases (Park \& Rogak, 2004), i.e., when growth tends to dominate over agglomeration. Going from case 3(c) to 3(b) and 3(a), the collision frequency $\beta_{0}$ decreases while keeping the surface growth rate constant (Table 3), which is equivalent to decreasing $\mathcal{R}_{\tau}$. This results in higher levels of $\epsilon_{E M D}$ for both 410 2-point and 3-point fixed-sectional methods, while $\epsilon_{E M D}$ remains relatively low and almost constant with the hybrid method (Table 5). Similarly, the error on the second moment $\epsilon_{M_{2}}$ increases from case 3(c) to 3(a), especially 
using the 2-point fixed-sectional method.

Using the same sections discretisation, the extra CPU cost of the hybrid method stays moderate (Table 5), between $8 \%$ and $42 \%$, except for cases $3(\mathrm{a})$ and 3(b) featuring parameters requiring sub-stepping to solve growth because of the small $\mathcal{R}_{\tau}$ ratios. Overall, the sectional method yields lower error as mesh is refined, but with a CPU cost rapidly growing. For instance, in the case $3(\mathrm{~d})$, where the grid of the sectional method has been refined to reach the accuracy of the hybrid method, the sectional method costs about 8 times more than the novel hybrid approach.

\section{Convergence and response to resolution parameters}

The proposed hybrid method features two resolution parameters: $N_{P}$ the total number of stochastic particles, and $M$ the number of sections discretizing the particle size space. The effects of these parameters on accuracy and CPU cost are now assessed. The CPU cost is normalised by the computational cost of the 3-point sectional method for $M=30$.

Test case 3(b) (growth and agglomeration) is chosen for this analysis with particle volume $v$ ranging from $6.66 \cdot 10^{-7}$ to $7.3 \cdot 10^{5}$. On the fixed-sectional part, geometric grids are used and $M$ and $F_{s}$ (Eq. (41)) are varied, keeping the size range constant. $N_{P}$ is varied for the stochastic part.

Comparison of the obtained solutions through the hybrid method and the sectional 3-point method is performed at the normalised time $t=7$. A measure of normalized $L_{1}$ error of calculated distributions against analytical solutions is provided. To make error comparison more meaningful across solutions obtained with different values of $M$, for each solution, the $M$ values of $N_{i} / \Delta v_{i}$ obtained after calculation are linearly interpolated over $M_{R}=$ 1000 logarithmically spaced $v_{i}$ points over the size range. Then, these $M_{R}$ calculated $N_{i} / \Delta v_{i}$ values are compared with the analytical $n\left(v_{i}\right)$ values. The error metric is defined as:

$$
L_{1_{\text {error }}}=\frac{\sum_{i=1}^{M_{R}}\left|N_{i} / \Delta v_{i}-n\left(v_{i}\right)\right| \cdot \Delta v_{i}}{\sum_{i=1}^{M_{R}} n\left(v_{i}\right) \cdot \Delta v_{i}} .
$$

Let us first examine the evolution of the normalized CPU cost as a function of $N_{P}$ and $M$. Figure 10 shows that from $N_{P}=10$ to $N_{P}=1000$, CPU cost is nearly constant for a given value of $M$. A slight increase is ob-

served from $N_{P}=1000$ to $N_{P}=10^{4}$ and then there is a sharp increase from 
Table 5: Accuracy and CPU costs. Case 1: Pure growth. Case 2: Pure agglomeration, Case 3: Growth/Loss \& agglomeration. Case 4: Nucleation \& growth.

\begin{tabular}{|c|c|c|c|c|}
\hline \multirow[t]{2}{*}{ Case } & \multirow[t]{2}{*}{ Error } & \multicolumn{3}{|c|}{ Method } \\
\hline & & Sectional 2pt & Sectional 3pt & Hybrid \\
\hline \multirow{4}{*}{$1(\mathrm{a})$} & $\overline{\epsilon_{M_{1}}(\%)}$ & -0.13 & - & -0.0078 \\
\hline & $\epsilon_{M_{2}}(\%)$ & 1.5 & - & -0.012 \\
\hline & $\epsilon_{E M D}$ & 0.11 & - & $1.9 \mathrm{e}-17$ \\
\hline & $\mathrm{CPU}$ time & 1.0 & - & 1.1 \\
\hline \multirow{3}{*}{$1(\mathrm{~b})$} & $\epsilon_{M_{1}}(\%)$ & -2.9 & -2.9 & -2.7 \\
\hline & $\epsilon_{M_{2}}(\%)$ & 770845 & 105 & -5 \\
\hline & CPU time & 1.0 & 1.0 & 1.4 \\
\hline \multirow{4}{*}{$2(\mathrm{a})$} & $\epsilon_{M_{1}}(\%)$ & 0.4 & 0.4 & -0.1 \\
\hline & $\epsilon_{M_{2}}(\%)$ & 1.6 & 1.6 & 0.7 \\
\hline & $\epsilon_{E M D}$ & 0.006 & 0.006 & 0.013 \\
\hline & CPU time & 1.0 & 1.0 & 1.3 \\
\hline \multirow{4}{*}{$2(\mathrm{~b})$} & $\overline{\epsilon_{M_{1}}(\%)}$ & -1.1 & -1.1 & -2.2 \\
\hline & $\epsilon_{M_{2}(\%)}$ & 0.4 & 0.4 & -1.4 \\
\hline & $\epsilon_{E M D}$ & 0.0008 & 0.0008 & 0.0048 \\
\hline & CPU time & 1.0 & 1.0 & 1.2 \\
\hline \multirow{4}{*}{$3(\mathrm{a})$} & $\overline{\epsilon_{M_{1}}(\%)}$ & 5.4 & 5.6 & -0.5 \\
\hline & $\epsilon_{M_{2}}(\%)$ & 48783 & 54 & 1.8 \\
\hline & $\epsilon_{E M D}$ & 0.62 & 0.19 & 0.06 \\
\hline & CPU time & 1.0 & 1.1 & 1.7 \\
\hline \multirow{4}{*}{$3(\mathrm{~b})$} & $\epsilon_{M_{1}}(\%)$ & 5.4 & 5.7 & 0.9 \\
\hline & $\epsilon_{M_{2}}(\%)$ & 22141 & 49 & 16 \\
\hline & $\epsilon_{E M D}$ & 0.60 & 0.17 & 0.10 \\
\hline & CPU time & 1.0 & 1.0 & 1.3 \\
\hline \multirow{4}{*}{$3(\mathrm{c})$} & $\overline{\epsilon_{M_{1}}(\%)}$ & 5.7 & 5.5 & 0.4 \\
\hline & $\epsilon_{M_{2}}(\%)$ & 9811 & 49 & 17 \\
\hline & $\epsilon_{E M D}$ & 0.57 & 0.16 & 0.13 \\
\hline & $\mathrm{CPU}$ time & 1.0 & 1.0 & 1.1 \\
\hline \multirow{4}{*}{$3(d)$} & $\overline{\epsilon_{M_{1}}(\%)}$ & 0.6 & 0.5 & 0.9 \\
\hline & $\epsilon_{M_{2}}(\%)$ & 776 & -1.4 & 16 \\
\hline & $\epsilon_{E M D}$ & 0.44 & 0.08 & 0.10 \\
\hline & CPU time & 1.0 & 1.1 & 0.1 \\
\hline \multirow{4}{*}{$3(\mathrm{e})$} & $\overline{\epsilon_{M_{1}}(\%)}$ & 5.4 & 5.8 & 1.1 \\
\hline & $\epsilon_{M_{2}}(\%)$ & 760 & 33 & 32 \\
\hline & $\epsilon_{E M D}$ & 0.47 & 0.24 & 0.09 \\
\hline & CPU time & 1.0 & 1.1 & 1.2 \\
\hline \multirow{4}{*}{4} & $\overline{\epsilon_{M_{1}}(\%)}$ & -3.4 & -3.4 & -3.4 \\
\hline & $\epsilon_{M_{2}}(\%)$ & 696121 & 60 & -9 \\
\hline & $\epsilon_{E M D}$ & 0.66 & 0.18 & 0.02 \\
\hline & CPU time & 1.0 & 1.0 & 1.5 \\
\hline
\end{tabular}


$N_{P}=10^{4}$ to $N_{P}=10^{5}$. This is because the calculation of agglomeration source terms is made at the level of the sections with a CPU cost scaling with $M$. On the other hand, stochastic particles need to be counted in each section and then reallocated over the sections according to the calculated agglomeration (and nucleation) source terms. The CPU cost of these operations depends on $M$ and $N_{P}$. In this test case, the cost linked to stochastic particles count and reallocation stays low as compared with the one linked to agglomeration source terms calculation up to $N_{P}=1000$ and becomes significant for $N_{P}=10000$ and $N_{P}=10^{5}$. Figure 11 confirms that CPU cost depends on $M$ for all values of $N_{P}$. From $N_{P}=10$ to $N_{P}=1000$ the CPU overhead linked to the use of the hybrid method instead of the sectional method is relatively low as compared with the overcost caused by an increase of $M$ (the lines are practically superposed at the plotted scale). The overcost stays moderate for $N_{P}=10000$ and turns very high for $N_{P}=10^{5}$.

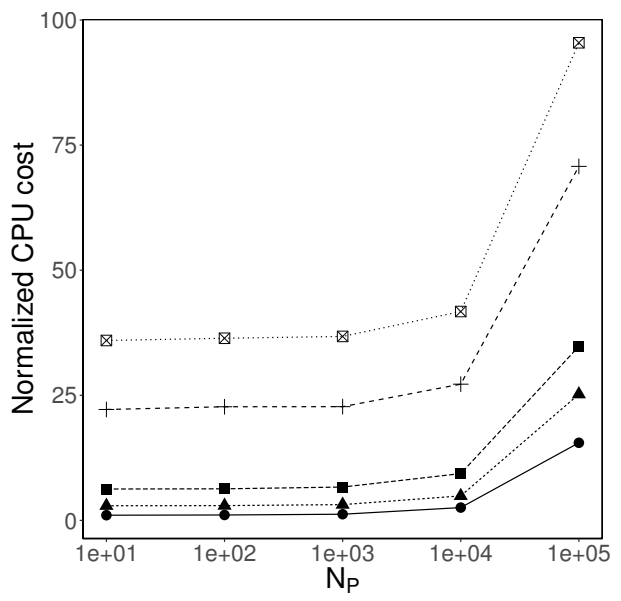

Figure 10: Normalized CPU cost vs $N_{P}$ for different values of $M$. Hybrid method. Solid line with full circles: $M=30$. Dotted line with triangles: $M=40$. Dashed line with full squares: $M=50$. Dashed line with plus symbols: $M=70$. Dotted line with cross-square symbols: $M=80$. Agglomeration and growth (Case 3, Table 3).

Now, the impact of $M$ and $N_{P}$ on accuracy is examined. Figure 12 shows that for $N_{P}=10$ and $N_{P}=100$ the hybrid method cannot perform well. After increasing $N_{P}$ up to 1000, the error on the PSD significantly decreases. However, for the tested values of $M$, further increasing $N_{P}$ only decreases $L_{1_{\text {error }}}$ marginally. Once a sufficient value of $N_{P}$ is reached, $M$ becomes the 455 main driver of $L_{1_{\text {error }}}$. 


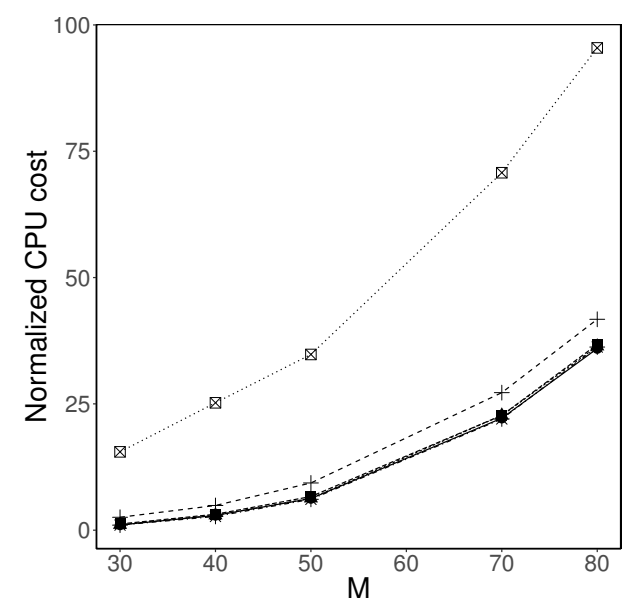

Figure 11: Normalized CPU cost vs $M$ for different values of $N_{P}$ (Hybrid method) and for the 3-point sectional method. Solid line with full circles: $N_{P}=10$. Dotted line with triangles: $N_{P}=100$. Dashed line with full squares: $N_{P}=1000$. Dashed line with plus symbols: $N_{P}=10000$. Dotted line with cross-square symbols: $N_{P}=10^{5}$. Solid line with stars: 3-point sectional. (Case 3, Table 3)

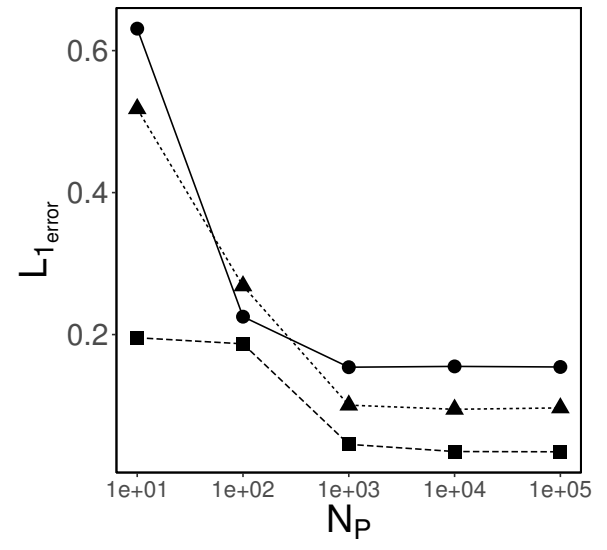

Figure 12: $L_{1_{\text {error }}}$ vs $N_{P}$ for different values of $M$ (Hybrid method). Solid line with full circles: $M=30$. Dotted line with triangles: $M=40$. Dashed line with full squares: $M=80$. (Case 3 , Table 3 at the normalised time $t=7$.)

Figure 13(a) shows that the error decreases with $M$ for both methods. Moreover, the hybrid method yields lower error than the 3-point sectional method for the whole range of $M$ tested. Figure 13(b) confirms that the hybrid method constitutes an interesting tradeoff between accuracy and CPU 


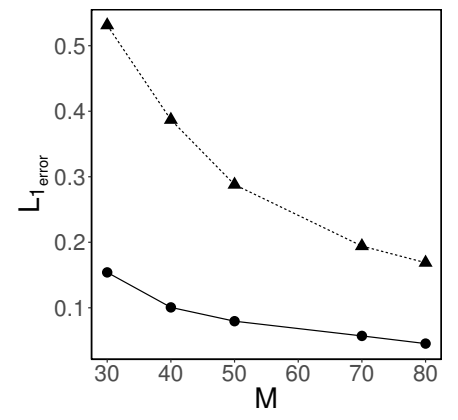

(a)

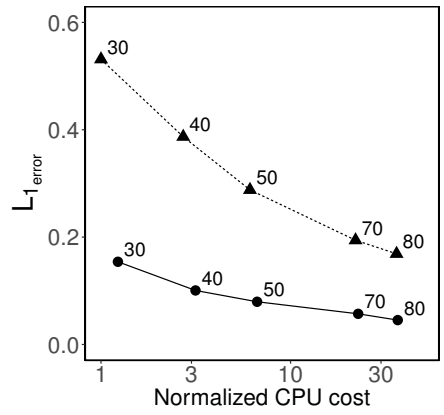

(b)

Figure 13: (a): $L_{1_{\text {error }}}$ vs $M$. (b): $L_{1_{\text {error }}}$ vs normalized CPU cost. Solid line with full circles: Hybrid method, $N_{P}=1000$. Dotted line with triangles: 3-point sectional method. Text labels in (b): $M$. (Case 3, Table 3 at the normalised time $t=7$.)

cost as compared with the 3-point sectional methods. Figure 14 illustrates the convergence of both 3-point sectional and hybrid method versus $M$.

Finally, the degree of variability of the solution induced by the random character of particle allocation is examined. Figure 15 shows average values and standard deviation over 10 runs of $n(v) \cdot v$ and of $v_{i}^{\star}$ (Eq. (32)) for case 3(b), with $M=40$ and $N_{P}=1000$. Non-negligible variance is observed, especially at the tails of the distribution where less stochastic particles are present. However, the distribution does not qualitatively vary from one run to the other. The observed standard deviation on $L_{1_{\text {error }}}$ over these 10 runs

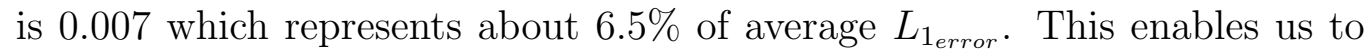
be confident on the level of accuracy of the solution obtained after only one run, without averaging over several runs (at least for $N_{P} \geq 1000$ ).

\section{Conclusion and perspectives}

A novel hybrid stochastic/sectional method for solving the population balance equation (PBE) has been presented. To design this numerical method, the particle size distribution (PSD) is decomposed into the total number density of particles times the particle size probability density function (PDF). The PDF is discretised into a fixed number of stochastic particles whose evolution is governed by a Monte Carlo procedure. The stochastic particles carry information on their size and the surface growth/loss is then solved in a direct manner, without resorting to any discretisation of the size space. This 


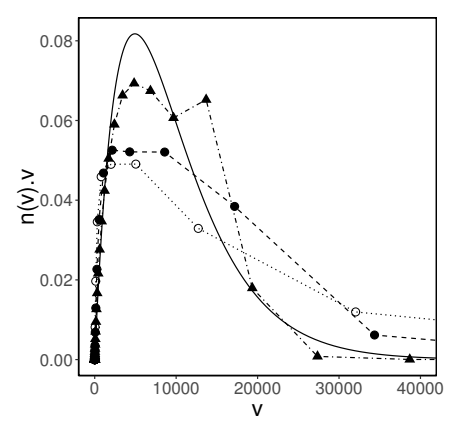

(a) Sectional

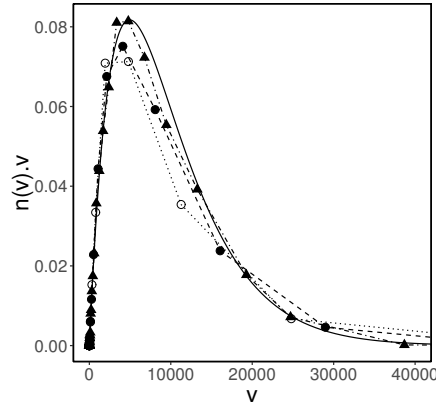

(b) Hybrid

Figure 14: Size distribution $n(v ; t) \cdot v$ for different values of $M$. (a): 3-point sectional method. (b): Hybrid method, $N_{P}=1000$. Solid line: analytical solution at $t=7$. Dashed-dot line with triangles: $M=80$. Dashed line with full circles: $M=40$. Dotted line with empty circles: $M=30$. (Case 3 , Table 3).

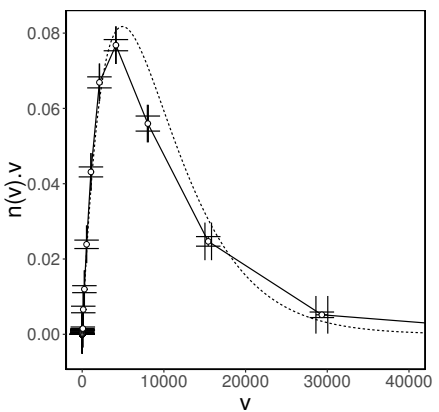

(a) lin-scale

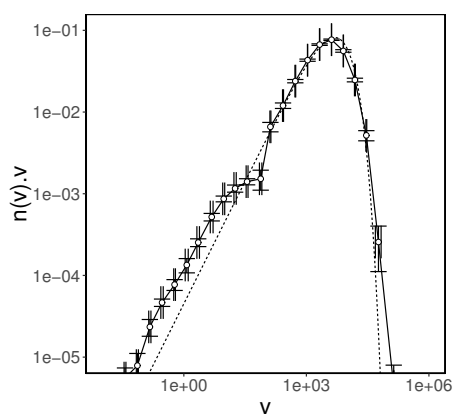

(b) log-scale

Figure 15: Average values and standard deviation over 10 runs. Dashed line: analytical solution at $\mathrm{t}=7.0$. Solid line with empty circles: hybrid method solution at $\mathrm{t}=7.0$ with $M=40$ and $N_{P}=1000$. Vertical error bars: standard deviation around average values of $n(v) \cdot v$. (Case 3, Table 3.) Horizontal error bars: standard deviation around average values of $v_{i}^{\star}$ (Eq. (32)). 
represents a serious advantage compared with fixed-sectional methods, which require some specific treatment to control the non-linear convective term representative of growth/loss in size space. These numerical treatments usually go with artificial diffusion of the PSD in size space.

In this hybrid approach, the agglomeration and nucleation sources are however computed following a fixed-sectional discretisation of sizes. These sources or sinks are then transformed into numbers of stochastic particles to be exchanged between sections following a Monte Carlo procedure. The transformation of these sources into integer numbers of particles leads to residuals, which are cumulated and transported through a usual 3-point fixedsectional approach, thus allowing for securing accuracy, even with a moderate number of stochastic particles and then at a moderate CPU cost.

The simulations of canonical test cases confirm the validity of the novel approach and allow for envisioning its application to three-dimensional flows. The stochastic particles would then need to be transported also in physical space, for instance following well-established flow transport methods within the context of Monte Carlo solutions. This may be done either in Lagrangian (Haworth, 2010) or Eulerian (Pope, 1981) contexts. In the Lagrangian formulation, aside from flow transport by a Langevin type equation, the time advancement of the PSD would be directly constructed from the set of stochastic particles present within a given mesh cell. In the Eulerian formulation, in additional to the local evolution of the stochastic particles simulating nucleation, surface growth/loss and agglomeration, the set of Monte Carlo particles fixed at every computational cell would exchange information with their neighboring nodes according to random processes simulating flow transport (Pope, 1981).

Another perspective lies in the possibility of transporting additional information within the stochastic particles, as a multivariate description, to improve the representation of particle physics.

\section{Acknowledgements}

The authors gratefully acknowledge the financial support of the European Union under the project SOPRANO, Horizon 2020 Grant Agreement No. 690724. The authors also benefited from discussion with Nicolas Perret and Matthieu Oullion from SOLVAY R\&I. 


\section{Appendix A.}

Two well-established 2- and 3-point discretisations of fixed-sectional methods are used to solve for surface growth/loss (Park \& Rogak, 2004). In the 2-point method, the choice is made to assign the grown particles to two adjacent sections conserving number of particles and mass. This technique, equivalent to so-called 'upwind' schemes, ensures stability at the cost of additional numerical diffusion. The 2-point discretisation reads:

$$
\frac{\partial N\left(v_{i}, t\right)}{\partial t}=\frac{G\left(v_{i-1}\right) N\left(v_{i-1}, t\right)}{v_{i}-v_{i-1}}-\frac{G\left(v_{i}\right) N\left(v_{i}, t\right)}{v_{i+1}-v_{i}}
$$

To reduce numerical diffusion, 3-point discretisations were discussed in the litterature (Hounslow et al., 1988; Park \& Rogak, 2004),

$$
\begin{aligned}
\frac{\partial N\left(v_{i}, t\right)}{\partial t} & =\frac{A_{i-1} G\left(v_{i-1}\right) N\left(v_{i-1}, t\right)}{v_{i-1}}+\frac{B_{i} G\left(v_{i}\right) N\left(v_{i}, t\right)}{v_{i}} \\
& +\frac{C_{i+1} G\left(v_{i+1}, t\right) N\left(v_{i+1}, t\right)}{v_{i+1}}
\end{aligned}
$$

The coefficients $A_{i}, B_{i}$ and $C_{i}$ are then calculated from PSD moments conservation equations, with a dependence on the slope of $d \ln \left(N\left(v_{i}, t\right)\right) / d \ln \left(v_{i}\right)$, to secure a reasonable balance between accuracy and stability, see Park \& Rogak (2004) for details. Time stepping is with a first-order forward Euler method.

\section{References}

Aubagnac-Karkar, D., Bakali, A. E., \& Desgroux, P. (2018). Soot particles inception and PAH condensation modelling applied in a soot model utilizing a sectional method. Combustion and Flame, 189, $190-206$.

Balthasar, M., \& Kraft, M. (2003). A stochastic approach to calculate the particle size distribution function of soot particles in laminar premixed flames. Combustion and Flame, 133, 289 - 298.

530

Das, S. K. (2016). A new turbulence-induced theoretical breakage kernel in the context of the population balance equation. Chemical Engineering Science, 152, $140-150$. 
Desgroux, P., Faccinetto, A., Mercier, X., Mouton, T., Karkar, D. A., \& Bakali, A. E. (2017). Comparative study of the soot formation process in a nucleation and a sooting low pressure premixed methane flame. Combust. Flame, 184, $153-166$.

Dopazo, C. (1979). Relaxation of initial probability density functions in the turbulent convection of scalar fields. Phys. Fluids, 22, 20-30.

Dopazo, C., Valino, L., \& Fuego, F. (1997). Statistical description of the turbulent mixing of scalar fields. International Journal of Modern Physics $B, 11$.

Eberle, C., Gerlinger, P., \& Aigner, M. (2017). A sectional PAH model with reversible PAH chemistry for CFD soot simulations. Combustion and Flame, 179, $63-73$.

Falope, G. O., Jones, A. G., \& Zauner, R. (2001). On modelling continuous agglomerative crystal precipitation via Monte Carlo simulation. Chemical Engineering Science, 56, $2567-2574$.

Ferziger, J. H., \& Perić, M. (1996). Computational Methods for Fluid Dynamics. Springer.

Filbet, F., \& Laurenot, P. (2004). Numerical simulation of the smoluchowski coagulation equation. SIAM Journal on Scientific Computing, 25, 20042028.

Fox, R. O. (2003). Computational models for turbulent reacting flows. Cambridge University Press.

Franzelli, B., Vié, A., \& Darabiha, N. (2019). A three-equation model for the prediction of soot emissions in LES of gas turbines. Proceedings of the Combustion Institute, 37, 5411-5419.

Frenklach, M. (2002). Method of moments with interpolative closure. Chemical Engineering Science, 57, 2229 - 2239.

Gelbard, F., \& Seinfeld, J. H. (1978). Numerical solution of the dynamic equation for particulate systems. Journal of Computational Physics, 28, $357-375$. 
Haworth, D. C. (2010). Progress in probability density function methods for turbulent reacting flows. Progress in Energy and Combustion Science, 36, $168-259$.

Hounslow, M. J., Ryall, R. L., \& Marshall, V. R. (1988). Discretized population balance for nucleation, growth, and aggregation. AIChE Journal, 34, $1821-1832$.

Hu, M., Jiang, X., \& Absar, M. (2018). Equivalence testing of complex particle size distribution profiles based on earth movers distance. AAPS $J, 20$.

Hulburt, H., \& Katz, S. (1964). Some problems in particle technology: A statistical mechanical formulation. Chemical Engineering Science, 19, 555 -574 .

Kollmann, W. (1990). The pdf approach to turbulent flow. Theoretical and Computational Fluid Dynamics, 1, 349-285.

Kumar, S., \& Ramkrishna, D. (1996a). On the solution of population balance equations by discretization - I: A fixed pivot technique. Chemical Engineering Science, 51, $1311-1332$.

Kumar, S., \& Ramkrishna, D. (1996b). On the solution of population balance equations by discretization - II: A moving pivot technique. Chemical Engineering Science, 51, 1333 - 1342.

Kumar, S., \& Ramkrishna, D. (1997). On the solution of population balance equations by discretization - III: Nucleation, growth and aggregation of particles. Chemical Engineering Science, 52, 4659 - 4679. Festshrift for Professor M. M. Sharma.

Lee, K. F., Patterson, R. I., Wagner, W., \& Kraft, M. (2015). Stochastic weighted particle methods for population balance equations with coagulation, fragmentation and spatial inhomogeneity. Journal of Computational Physics, 303, 1-18.

Leung, K., Lindstedt, R., \& Jones, W. (1991). A simplified reaction mechanism for soot formation in nonpremixed flames. Combustion and Flame, $87,289-305$. 
Li, T. S., Livk, I., \& Ilievski, D. (2001). The influence of crystalliser configuration on the accuracy and precision of gibbsite crystallisation kinetics estimates. Chemical Engineering Science, 56, 2511 - 2519.

Lindstedt, R. P., \& Louloudi, S. A. (2005). Joint-scalar transported PDF modeling of soot formation and oxidation. Proceedings of the Combustion Institute, 30, 775-783.

Lister, J. D., Smit, D. J., \& Hounslow, M. J. (1995). Adjustable discretized population balance to growth and aggregation. AIChE Journal, 41, $591-$ 603.

Lundgren, T. S. (1967). Distribution function in the statistical theory of turbulence. Physics Fluids, 10, 969-975.

Ma, G., Wen, J. Z., Lightstone, M. F., \& Thomson, M. J. (2005). Optimization of soot modeling in turbulent nonpremixed ethylene/air jet flames. Combustion Science and Technology, 177, 1567-1602.

Marchisio, D. L., \& Fox, R. O. (Eds.) (2007). Multiphase reacting flows: Modelling and simulation volume 492 of CISM International Center for Mechanical Sciences. Wien: Springer-Verlag.

Mueller, M., Blanquart, G., \& Pitsch, H. (2009). Hybrid method of moments for modeling soot formation and growth. Combustion and Flame, 156, $1143-1155$.

Nguyen, T., Laurent, F., Fox, R., \& Massot, M. (2016). Solution of popu615 lation balance equations in applications with fine particles: Mathematical modeling and numerical schemes. Journal of Computational Physics, 325, $129-156$.

Oullion, M., Reynolds, G. K., \& Hounslow, M. J. (2009). Simulating the early stage of high-shear granulation using a two-dimensional Monte-Carlo approach. Chemical Engineering Science, 64, 673-685.

Park, S., \& Rogak, S. (2004). A novel fixed-sectional model for the formation and growth of aerosol agglomerates. Journal of Aerosol Science, 35, 1385 -1404 . 
Patterson, R. I., \& Kraft, M. (2007). Models for the aggregate structure of soot particles. Combustion and Flame, 151, 160 - 172.

Patterson, R. I., Wagner, W., \& Kraft, M. (2011). Stochastic weighted particle methods for population balance equations. Journal of Computational Physics, 230, $7456-7472$.

Pesmazoglou, I., Kempf, A. M., \& Navarro-Martinez, S. (2016). Stochastic modelling of particle aggregation. International Journal of Multiphase Flow, 80, $118-130$.

Pope, S. (1981). Monte Carlo method for the PDF equations of turbulent reacting flow. Combust. Sci. Tech., 25, 159-174.

Qamar, S., Ashfaq, A., Warnecke, G., Angelov, I., Elsner, M., \& SeidelMorgenstern, A. (2007). Adaptive high-resolution schemes for multidimensional population balances in crystallization processes. Computers and Chemical Engineering, 31, 1296 - 1311.

Ramabhadran, T. E., Peterson, T. W., \& Seinfeld, J. H. (1976). Dynamics of aerosol coagulation and condensation. AIChE Journal, 22, 840-851.

Ramkrishna, D. (1985). The status of population balances. Reviews in Chemical Engineering, 3, 49.

Ramkrishna, D. (2000). Population balances. Theory and applications to particulate systems in engineering. Academic Press San Diego.

Rigopoulos, S., \& Jones, A. G. (2003). Finite-element scheme for solution of the dynamic population balance equation. AIChE Journal, 49, 1127 1139 .

Rodrigues, P., Franzelli, B., Vicquelin, R., Gicquel, O., \& Darabiha, N. (2018). Coupling an LES approach and a soot sectional model for the study of sooting turbulent non-premixed flames. Combustion and Flame, $190,477-499$.

Rubner, Y., Tomasi, C., \& Guibas, L. J. (1998). A metric for distributions with applications to image databases. In Sixth International Conference on Computer Vision (IEEE Cat. No.98CH36271) (pp. 59-66). 
Salenbauch, S., Hasse, C., Vanni, M., \& Marchisio, D. L. (2019). A numerically robust method of moments with number density function reconstruction and its application to soot formation, growth and oxidation. $J$. Aerosol Sci., (pp. 34-49).

Schiener, M., \& Lindstedt, R. (2019). Transported probability density function based modelling of soot particle size distributions in non-premixed turbulent jet flames. Proceedings of the Combustion Institute, 37, 10491056.

Scott, W. T. (1968). Analytic studies of cloud droplet coalescence I. Journal of the Atmospheric Sciences, 25, 54-65.

Sewerin, F., \& Rigopoulos, S. (2017). An explicit adaptive grid approach for the numerical solution of the population balance equation. Chemical Engineering Science, 168, 250 - 270.

Smith, M., \& Matsoukas, T. (1998). Constant-number monte carlo simulation of population balances. Chemical Engineering Science, 53, 1777 - 1786.

Smoluchowski, M. (1917). Mathematical theory of the kinetics of the coagulation of colloidal solutions. Zeitschrift fr Physikalische Chemie, 19, 129-135.

Solsvik, J., \& Jakobsen, H. A. (2015). The foundation of the population balance equation: A review. Journal of Dispersion Science and Technology, $36,510-520$.

Tsantilis, S., Kammler, H. K., \& Pratsinis, S. E. (2002). Population balance modeling of flame synthesis of titania nanoparticles. Chemical Engineering Science, 57, 2139-2156.

Urbanek, S. (2012). Package emdist. URL: https://cran.r-project.org/ web/packages/emdist/emdist.pdf.

Zhao, H., \& Zheng, C. (2013). A population balance-monte carlo method for particle coagulation in spatially inhomogeneous systems. Computers and Fluids, 71, 196-207.

Zucca, A., Marchisio, D. L., Barresi, A. A., \& Fox, R. O. (2006). Implementation of the population balance equation in CFD codes for modelling soot 685 formation in turbulent flames. Chemical Engineering Science, 61, 87 - 95. 